\title{
The subacute inhalation toxicity of 109 industrial chemicals
}

\author{
J. C. GAGE
}

Imperial Chemical Industries Limited, Industrial Hygiene Research Laboratories, Alderley Park, Macclesfield, Cheshire

Gage, J. C. (1970). Brit. J. industr. Med., 27, 1-18. The subacute inhalation toxicity of 109 industrial chemicals. The inhalation toxicity of 109 substances has been studied by exposing experimental animals to known concentrations in air for periods of about three weeks. The toxic properties of these substances are reviewed in relation to the effects of similar compounds on animals and on man. Provisional operational limits are suggested to assist in the design of new plant and in the establishment of codes for safe manufacturing practice.

Most serious occupational diseases arising from exposure to chemicals are caused by an attack on, or absorption through, the respiratory tract. On occasion such effects can be predicted from oral or parenteral administration of the chemical to experimental animals, but, in general, if a substance presents an inhalation risk, it is preferable to undertake a direct investigation by exposing animals to known concentrations in air. This survey covers the work on inhalation toxicity which has been undertaken over a period of 20 years in a laboratory engaged in the study of the toxic properties of chemicals used in industry.

All of the samples investigated for inhalation toxicity over this period were submitted by the manufacturing divisions of ICI Ltd.; they averaged about 30 a year. Not all of these have been included in this survey. Some were of too indeterminate a composition, such as still residues; some were proprietary products whose formulation was uncertain; for some the investigation was never completed for a variety of reasons; for a few, publication has been restricted for commercial or other considerations.

The aim of thes investigations has been to provide information to aid in plant design and in the establishment of safety precautions to prevent occupational disease when the materiais are produced or used in manufacturing operations. The experimental results have led to a decision on whether the compound may be handled without special precautions other than those demanded by sound manufacturing practice, whether exhaust ventilation should be installed or whether other features in plant design are required to prevent excessive exposure. The results have also enabled a prediction of the effects likely to be encountered in man from brief or repeated over-exposure, and have provided guidance on treatment to a works medical officer confronted with an accident or with a failure to apply the recommended safety precautions.

The compounds investigated are indexed below in alphabetical order.

1-Acetyl- $\gamma$-butyrolactone, 10

Acrylic acid, 6

Acrylyl chloride, 14

Adipic acid, 6

2-Aminobutan-1-ol, 10

2-Aminomethyl-3, 4-dihydropyran, 11

Bis-2-chloro-1-methylethyl ether, 6

Bis-2-ethoxyethyl ether, 6

2,2-Bis-p-hydroxyphenylpropane, 14

Bis-2-methoxyethyl ether, 6

Bis-3-methylbutyl peroxydicarbonate 11

Bispentafluorosulphur oxide, 13

(2-Bromoethoxy) benzene, 7

5-Bromopentan-2-one, 6 
2-t-Butoxyethanol, 5

s-Butylamine, 9

t-Butyl peracetate, 11

t-Butyl peroxypivalate, 11

n-Butyraldehyde, 6

Cetostearyl methacrylate, 8

Chloroacetonitrile, 10

6-Chlorododecafluorohexylsulphur pentafluoride, 14

2-Chloroethylsulphur pentafluoride, 13

1-Chloronaphthalene (technical), 8

4-Chloro-octafluorobutylsulphur pentafluoride, 13

Chloropentafluorobenzene, 7

1-Chloropropan-2-ol, 5

2-Chloropropane, 6

2-Chlorotetrafluoroethylsulphur pentafluoride, 13

Cumene a-hydroperoxide, 11

$\mathrm{N}$-2-Cyanoethylaniline, 10

Decahydronaphthalene, 5

1,6-Diaminohexane, 9

Dichlorobutenes (mixed isomers), 7

1,1-Dichloroethene, 7

1,3-Dichlorotetrafluorobenzene, 7

$a$ - Dicyclopentadiene, 4

1-Diethylaminopentan-2-one, 10

Diethylenetriamine, 10

$00^{\prime}$-Diethyl phosphorochloridothionate, 12

Dimethoxymethane, 5

2-Dimethylaminoethyl methacrylate, 8

3,6-Dimethyl-1,2-benzisoxazole, 11

Dimethyl carbonate, 9

Dimethyl disulphide, 13

3,5-Dimethylmorpholine, 11

Dinonylamine, 9

Diphenyldimethoxysilane, 12

Dipropionyl peroxide, 11

Di-s-butylamine, 9

Divinyl disulphide, 13

Dixylyl disulphide, 13

Ethyl chloroformate, 9

Ethyl 3-chlorophenylformimidate, 14

2-Ethylhexyl acrylate, 8

2-Ethylhexyl methacrylate, 8

2-Ethyl-2-hydroxymethylpropane-1, 3-diol, 5

Ethyl t-butyl peroxyoxalate, 11

$N$-Formylpiperidine, 10

Glycol dimethacrylate, 8

Hexachlorobutadiene, 7

Hexafluorobenzene, 7

$N N$-Hexamethyleneadipamide, 14

2-Hydroxyethyl methacrylate, 8

2-Hydroxypropyl methacrylate, 8

4-Hydroxytetrahydropyran, 11

Iron pentacarbonyl, 14

Isobutoxyethene, 5

Isobutyraldehyde, 6

Iso-octanol, 5

2-Isopropoxyethanol, 5

Isopropyl chloroformate, 9

Lauryl mercaptan, 13

Lauryl methacrylate, 8

Methacrylic acid, 6

2-Methoxy-3,4-dihydropyran, 11

Methoxyethene, 5

2-Methylbenzoxazole, 10
2-Methylbuta-1,3-diene, 4

Methyl chloroformate, 8

2-Methyl-1,3-dioxolan, 10

Methyl isothiocyanate, 8

Methyl nitrite, 9

Methyl salicylate, 8

2-Methylthiazole, 10

Nonylamine, 9

Octyl methacrylate, 8

Pentachloropyridine, 10

Phosphorus tri-isocyanate, 12

Propionaldehyde, 6

n -Propyl cyanide, 10

$N$-Propylethylideneamine, 14

Silicon tetrafluoride, 12

Silicon tetraisocyanate, 12

Sulphur chloride pentafluoride, 13

Sulphur dichloride, 12

3a,4,7,7a-Tetrahydro- 4,7-methanoindene, 4

Tetramethylsilane, 12

Tributylamine, 9

Tributyl phospite, 12

1,2,4-Trichlorobenzene, 7

Trichloromethylsulphenyl chloride, 13

1,3,5-Trichlorotrifluorobenzene, 7

Trimethoxyboroxine, 14

1, 2, 4-Trimethylbenzene, 5

Trinonylamine, 9

Trispentafluoroethyl)methanol, 5

1, 1, 1-Trishydroxymethylpropane bicyclic phosphite, 12 Vinyl acetate, 8

Vinylsulphur pentafluoride, 13

\section{Methods and materials}

Samples investigated

The samples submitted by ICI Divisions were prepared in either the Research Department or an experimental plant, or were taken from full-scale production. The size of the sample available determined to some extent the scale of the experimental work. Few of the samples could be described as pure chemicals; they were not fractionated before use as the toxicological properties of the materials as supplied were relevant to these investigations. Where the sample was known to contain a considerable admixture of other components, this information is included in the Results section.

\section{Design of exposure chambers}

In all of these experiments the animals have been exposed to dynamic atmospheres, that is, to atmospheres continuously generated and passed through the exposure chamber. The design of exposure chamber has varied with the number of animals involved and with the nature of the substance under investigation. For groups of four or fewer rats a glass desiccator, containing wire mesh partitions to separate the animals, was used. Larger numbers, up to eight rats, were exposed in the chamber described elsewhere (Gage, 1959); usually the inner Perspex chamber of that design was replaced by a glass cylinder, $30 \mathrm{~cm}$ diameter and $25 \mathrm{~cm}$ high. For atmospheres containing particulate matter a chamber with a more pyramidal top (Gage, 1968) was used. 
Generation of the test atmospheres

The air used for the atmospheres was filtered, dried to a relative humidity of less than $10 \%$, and supplied at a line pressure of $1 \mathrm{~atm}\left(1.013 \times 10^{5} \mathrm{Nm}^{-2}\right)$. In the Results section, the methods used to prepare the atmospheres are indicated by letters in parentheses which refer to the following list. The letter is followed by any information relating to the particular experiment.

A A nearly saturated vapour obtained by passing air through a liquid contained in a bubbler with a sintered glass air-distributor disc. The volume of the liquid was usually $10-20 \mathrm{ml}$ and, if the size of the sample available permitted, it was replaced daily. Unless otherwise stated, the bubbler was maintained in a water-bath at room temperature, about $20^{\circ} \mathrm{C}$.

B A nearly saturated vapour obtained by passing air through a column of a granular solid. If the sample supplied was a fine powder, it was dispersed on the suiface of granular kieselguhr.

C An atmosphere prepared by methods A or B and diluted to a known extent with clean air.

D A vapour concentration by injecting a liquid at a known rate into a metered stream of air by means of a controlled fluid-feed atomizer (Gage, 1953). For concentrations much less than $100 \mathrm{ppm}$ a solution of the liquid in a toxicologically inert solvent was used. For very volatile liquids the syringe was cooled in an ice-water bath.

E A metered stream of a gas or vapour from a cylinder was diluted with a metered stream of air. The diluted gas was passed through a jet to produce efficient mixing by turbulence.

F A gas or vapour contained in a large polyethylene bag at atmospheric pressure was intı oduced into a metered air stream at a known rate by means of a peristaltic pump (Watson Marlow).

G A powdered solid injected into a metered air stieam at a known rate by the apparatus described by Byers and Gage (1961).

H A condensed fume prepared by passing an aerosol obtained by methods $\mathrm{D}$ or $\mathrm{G}$ through a well-insulated cylindrical electric furnace located in the central tube of the exposure chamber. The furnace was heated to a temperature sufficiently high to volatilize the substance, which on cooling condensed to a fume.

I A method was devised for the continuous generation of methyl nitrite, which is too unstable to be isolated and stored. A solution of hydrochloric acid in methanol was injected at a known rate on to a mixture of equal parts of sodium nitrite and anhydrous sodium sulphate. In the presence of excess methanol the rate of addition of hydrochloric acid defined the rate of liberation of methyl nitrite. The vapour diffused through a sintered glass plate into a metered air stream.

\section{Measurement of concentration}

The nearly saturated concentration prepared by methods $A$ and $B$ was estimated by weighing the sample before and after the day's run, and relating the weight loss to the volume of air passing. This concentration, expressed in milligrammes per litre, was converted to parts per million on the assumption that the sample was pure.
Both of these estimates are only approximate; this applies particularly to materials of low volatility and to impure samples when the more volatile fraction may evaporate first. For these reasons method D was usually preferred for such materials.

With the other methods the concentrations have usually been those calculated from the known rate of introduction of the substance into the air stream. In some experiments the concentration was checked by direct analysis of the atmosphere and was found to be within $10 \%$ of the expected value. A direct determination was always made when the method could not give a reliable indication of concentration, as with aerosols or organic peroxides. Concentrations determined by analysis are indicated by a superscript letter which refers to the following list.

a Gas-liquid chromatography. A Pye 104 instrument with a flame ionization detector was used. The column was $5 \mathrm{ft}$ by $\frac{1}{4}$ in, filled with $60-80$ mesh Celite coated with SE30. The temperature of the column was maintained at about $20 \%$ below the boiling point of the liquid. A sample of the atmosphere was injected directly into the $\mathrm{N}_{2}$ carrier gas by means of a gas sampling valve $(0 \cdot 5-10 \mathrm{ml})$.

b Peroxides by iodometry. A measured volume of the air was passed through a $1 \% \mathrm{w} / \mathrm{v}$ aqueous potassium iodide solution, and the liberated iodine was measured absorptiometrically at $425 \mathrm{~nm}$.

c Peroxides by oxidation of ferrous thiocyanate. A $50-\mathrm{ml}$ sample of the air was collected in a large glass syringe containing $10 \mathrm{ml}$ ferrous thiocyanate reagent $(100 \mathrm{ml} 0.5 \% \mathrm{w} / \mathrm{v}$ ammonium thiocyanate, $1 \mathrm{ml} 6 \mathrm{~N}$ sulphuric acid, $0 \cdot 1 \mathrm{~g}$ ferrous ammonium sulphate, 100 $\mathrm{ml}$ water, prepared freshly each day). The syringe was shaken for 5 minutes and the ferric thiocyanate concentration was measured absorptiometrically at $460 \mathrm{~nm}$.

d Titration. A measured volume of the air was passed through water (sulphur dichloride) or $10 \% \mathrm{v} / \mathrm{v}$ aqueous ethanol (adipic acid) and the absorbed acid was titrated with sodium hydroxide solution.

e Methyl nitrite. A measured volume of the air was passed through $10 \mathrm{ml}$ reagent $(0 \cdot 1 \mathrm{~g}$ chloroaniline, $10 \mathrm{ml} \mathrm{N} \mathrm{HCl}$, acetic acid to 1 litre); $2 \mathrm{ml} 1 \%$ aqueous $N$-(1-naphthyl)ethylenediamine dihydrochloride was added, and after 15 minutes the absorbance was measured at $550 \mathrm{~nm}$.

\section{Design of the experiments}

Alderley Park specific-pathogen-free rats with an average weight of $200 \mathrm{~g}$ were used in most of these experiments. They were maintained in the exposure chamber for periods of up to 6 hours, and between repeated daily exposures they were returned to their cages where food and water were freely available. In the initial experiments the concentrations were selected to produce, if possible, acute effects after short exposures. Thereafter the exposure period was extended and the concentration lowered until the animals could survive 6-hour exposures, five days a week, for up to four weeks. With liquids, the vapour pressure limited the range of concentrations which could be tested in these acute and subacute experiments. The rats were weighed each morning, and their conditions and behaviour were recorded throughout the exposure period. Urine was collected overnight 
after the last exposure day for biochemical tests.: The animals were left overnight with food and drink. On the following day the rats were anaesthetized with halothane and partially exsanguinated by heart puncture for haematological tests. After a gross examination of the organs, the lungs were inflated with formol-saline and immersed in the same fixative. The following organs were also taken for microscopical examination after fixation in formol-corrosive: lungs, liver, kidneys, spleen, and adrenals; and occasionally heart, jejunum, ileum, and thymus.

If at any stage effects were observed which could be attributed to the exposure, the experiment was repeated with progressively lower concentrations until a concentration was reached which was without effects on the animals. At intervals of about two months, batches of control rats were maintained in a chamber for the exposure period, in order to check the characteristics of the colony.

\section{Results}

\section{Presentation of experimental results}

Where practicable, the chemical name used is that recommended by the International Union of Pure and Applied Chemistry (IUPAC) classification. This is followed in brackets by any other name in common usage. Where the IUPAC name is little used, as with methacrylic acid, the widely used name appears first, followed by the systematic name. Any information on purity is included. A structural formula is given. The physical state and the melting or boiling points (where known) are stated.

Details of the experiments undertaken and the results obtained are presented according to the following scheme:

Atmospheric concentration and method of generation; number, sex, and species of animals; number and duration of exposures; clinical observations; autopsy.

The following annotations explain the conventions used in this presentation.

Atmosphere The measured or calculated concentration followed in parentheses by a letter indicating the method of generating the atmosphere and any special details. A superscript letter indicates the method used for determining the concentration. These letters refer to procedures detailed in the Methods section. Unless otherwise stated, the concentrations were dilutions of a vapour or a gas. With 'saturated' atmospheres, the concentration in brackets is that estimated from the weight loss, unless otherwise indicated.

Clinical observations The following descriptive terms have been used: nose irritation, sneezing progressing with increasing severity to a nasal discharge and a bloody exudate; eye irritation, eyes closed, progressing to lachrymation; respiratory difficulty, rapid shallow breathing progressing to laboured and slow breathing; lethargy, less than normal activity and a lower response to noise; hypersensitive or unresponsive, an increased or diminished reaction, respectively, to noise and handling; incoordination, unsteady movements, staggering gait; no toxic signs implies that the animals remained in good condition. In most experiments blood and urine tests were made at the lower concentrations, and any abnormal results are indicated. The urine tests included specific gravity, $p \mathbf{H}$, reducing sugars, bilirubin, and protein. The blood tests included haemoglobin (Hb) concentration, packed cell volume, mean corpuscular $\mathrm{Hb}$ content, a white and differential cell count, a platelet count, clotting function, and the concentration of urea sodium, and potassium. Control tests for the haematological examination were made on the group of animals before exposure. Autopsy-no comment on the gross pathology indicates that the organs appeared normal. Comment after (histol.) indicates effects seen on microscopical examination of the tissues. Organs normal indicates that these examinations revealed no changes which could be attributed to the treatment.

\section{Hydrocarbons}

2-Methylbuta-1,3-diene

[isoprene]

liq. b.p. $34^{\circ} \mathrm{C}$

$6000 \mathrm{ppm}(\mathrm{D}$, cooled): $2 \mathrm{M}$ 2F rats: $6 \times 6-\mathrm{hr}$ exposures: no toxic signs: autopsy, lungs slightly congested, (histol.) organs normal

1670 ppm (D, cooled): $2 \mathrm{M} 2 \mathrm{~F}$ rats: $15 \times 6$-hr exposures: no toxic signs: autopsy, organs normal

3a,4,7,7a-Tetrahydro-4,7-methanoindene [ $\alpha$-Dicyclopentadiene]

solid m.p. $25-30^{\circ} \mathrm{C}$, b.p. $170^{\circ}$

(decomp.)

2500 ppm(D, pet.ether): $2 \mathrm{M}$

$2 \mathrm{~F}$ rats: $1 \times 1$-hr exposure: eye and nose irri-

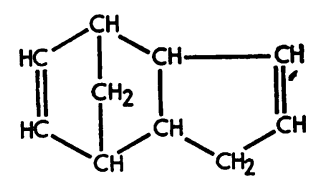

tation, dyspnoea, narcosis, 1 died later: autopsy (histal.) lungs, liver and kidneys congested

$1000 \mathrm{ppm}(\mathrm{D}$, pet. ether): $2 \mathrm{M}$ 2F rats: $1 \times 4-\mathrm{hr}$ exposure: eye and nose irritation, dyspnoea, muscular incoordination, tremors, hypersensitivity, all died later: autopsy, lungs congested, (histol.) lungs, liver, and kidneys congested

$250 \mathrm{ppm}$ (D, pet ether): $2 \mathrm{M} 2 \mathrm{~F}$ rats: $10 \times 6-\mathrm{hr}$ exposures: 1 died after 2nd exposure, survivors lost weight, nose irritation, dyspnoea, lethargic, tremors, hypersensitive, blood tests normal: autopsy, organs normal

100 ppm (D, pet ether): $4 \mathrm{M} 4 \mathrm{~F}$ rats: $15 \times 6-\mathrm{hr}$ exposures: no toxic signs: autopsy, organs normal 


\section{1,2,4-Trimethylbenzene \\ [pseudocumene] \\ liq. b.p. $169^{\circ} \mathrm{C}$ \\ Saturated (A) [10 mg/litre, $2000 \mathrm{ppm}]: 4 \mathrm{M} 4 \mathrm{~F}$ rats: $12 \times 6$-hr exposures: nose and eye irritation, respiratory difficulty, lethargy, tremors, low weigh increase, blood tests normal: autopsy, organs normal \\ $1000 \mathrm{ppm}(\mathrm{D}), 4 \mathrm{M}$ 4F rats: $15 \times 6$-hr exposures: initial slight eye and nose irritation, blood tests normal: autopsy, organs normal}

Decahydronaphthalene

[decalin]

$\mathrm{C}_{10} \mathrm{H}_{18}$

liq. b.p. $183-196^{\circ} \mathrm{C}$

$1000 \mathrm{ppm}$ (D): $4 \mathrm{M}$ 4F rats: $1 \times 4$-hr exposure: tremors, convulsions, 3 deaths: autopsy, lungs congested

$200 \mathrm{ppm}$ (D): $4 \mathrm{M}$ 4F rats: $20 \times 6$-hr exposures: no toxic signs: autopsy, organs normal

\section{Alcohols}

Iso-octanol (mixture of branched chain alcohols, approx. $\left.\mathrm{C}_{8} \mathrm{H}_{17} \mathrm{OH}\right)$

liq. b.p. $185-9^{\circ} \mathrm{C}$

Saturated (A) [1 mg/litre, $180 \mathrm{ppm}$ ]: $3 \mathrm{~F}$ rats: $13 \times 6-\mathrm{hr}$ exposures: no toxic signs: autopsy, organs normal

\section{2-Is opropoxyethanol}

[isopropyl glycol ether]

liq. b.p. $140-144^{\circ} \mathrm{C}(90 \%)$

$1000 \mathrm{ppm}$ (D): $4 \mathrm{M}$ 4F rats: $15 \times 6-\mathrm{hr}$ exposures: initial nasal irritation, lethargy, haemoglobinuria, porphyrinuria, $\mathrm{Hb}$ low, (4th day) MCHC low, reticulocytosis, later blood and urine normal: autopsy, organs appeared normal, (histol.) lungs congested

$300 \mathrm{ppm}$ (D): $4 \mathrm{M}$ 4F rats: $15 \times 6-\mathrm{hr}$ exposures: slight transient fall in $\mathrm{Hb}$ and MCHC (3rd day): autopsy (histol.) lungs congested

$100 \mathrm{ppm}$ (D): $4 \mathrm{M}$ 4F rats: $15 \times 6-\mathrm{hr}$ exposures: no toxic signs: autopsy, organs normal

2-t-Butoxyethanol

[t-butyl glycol ether]

liq. b.p. $152^{\circ} \mathrm{C}$

Saturated (A) [13 mg/litre, $2400 \mathrm{ppm}]: 4 \mathrm{M}$ 4F rats: $1 \times 5-\mathrm{hr}$ exposure: comatose, haematuria, $\mathrm{Hb}$ $35-50 \%$ normal, all died $1-2$ days later

$250 \mathrm{ppm}$ (D): $4 \mathrm{M}$ 4F rats: $4 \times 6-\mathrm{hr}$ exposures: initial haemaglobinuria and lethargy, low $\mathrm{Hb}$ and MCHC, weight loss

$100 \mathrm{ppm}(\mathrm{D}): 4 \mathrm{M} 4 \mathrm{~F}$ rats: $15 \times 6-\mathrm{hr}$ exposures: no toxic signs, urine and blood tests normal apart from increased red cell osmotic fragility: autopsy, organs normal

$50 \mathrm{ppm}(\mathrm{D}): 4 \mathrm{M} 4 \mathrm{~F}$ rats: $15 \times 6-\mathrm{hr}$ exposures: as $100 \mathrm{ppm}$ experiment

$20 \mathrm{ppm}$ (D): $4 \mathrm{M}$ 4F rats: $15 \times 6$-hr exposures: no toxic signs, blood normal: autopsy, organs normal

Tris(pentafluoroethyl)methanol

$\left(\mathrm{C}_{2} \mathrm{~F}_{5}\right)_{3} \mathrm{C} \cdot \mathrm{OH}$

[perfluorotriethylcarbinol]

liq. b.p. $105^{\circ} \mathrm{C}$
$100 \mathrm{ppm}(\mathrm{D}): 4 \mathrm{M}$ 4F rats: $1 \times 1$-hr exposure: gasping respiration, nasal discharge, 7 rats died, rapid rigor

$25 \mathrm{ppm}$ (D, pet. ether): 4M 4F rats: $2 \times 6-\mathrm{hr}$ exposures: gasping, nasal discharge, 1 died: autopsy, lungs haemorrhagic

$10 \mathrm{ppm}(\mathrm{D}$, pet. ether): $4 \mathrm{M} 4 \mathrm{~F}$ rats: $4 \times 6-\mathrm{hr}$ exposures: increased water consumption, rectal temp. $39 \cdot 5^{\circ} \mathrm{C}$, nasal discharge, rapid breathing, prostrate, blood tests normal, 2 died: autopsy, lungs haemorrhagic

$2.5 \mathrm{ppm}$ (D, pet. ether): $4 \mathrm{M}$ F4 rats: $15 \times 6-\mathrm{hr}$ exposures: increased water consumption, prostrate, blood and urine tests normal, weight increase normal: autopsy, organs normal

$1.0 \mathrm{ppm}$ (D, pet. ether): $4 \mathrm{M} 4 \mathrm{~F}$ rats: $15 \times 6-\mathrm{hr}$ exposures: no toxic signs: autopsy, organs normal

1-Chloropropan-2-ol

[propylene chlorohydrin]

liq. b.p. $133^{\circ} \mathrm{C}$

$1000 \mathrm{ppm}$ (D): $2 \mathrm{M} 2 \mathrm{~F}$ rats: $2 \times 6$-hr exposures: lethargy after 1st exposure, 3 days later given 2nd exposure, 1 died: autopsy, lungs oedematous and congested, liver pale, (histol.) lungs interstitial inflammatory exudate, liver cells swollen and vacuolated with nuclear degeneration

$250 \mathrm{ppm}^{\mathrm{a}}$ (D): $2 \mathrm{M} 2 \mathrm{~F}$ rats: $15 \times 6-\mathrm{hr}$ exposures: lethargy, irregular weight gain, blood and urine tests normal: autopsy (histol.) lungs congested with perivascular oedema

$100 \mathrm{ppm}$ (D): $4 \mathrm{M}$ 4F rats: $15 \times 6$-hr exposures: no toxic signs: autopsy (histol.) lungs congested with perivascular oedema

$30 \mathrm{ppm}^{2}$ (D, ethanol): $4 \mathrm{M}$ 4F rats: $14 \times 6$-hr exposures: no toxic signs: organs normal

2-Ethyl-2-hydroxymethylpropane-1, 3-diol

[Trishydroxymethylpropane] $\quad \mathrm{C}_{2} \mathrm{H}_{5} \cdot \mathrm{C}\left(\mathrm{CH}_{2} \mathrm{OH}\right)_{3}$ solid m.p. $58^{\circ} \mathrm{C}$, b.p. ca. $150^{\circ} \mathrm{C}(0 \cdot 2-0 \cdot 4 \mathrm{~mm})$

Saturated $\left(\mathrm{A}, 70^{\circ} \mathrm{C}\right)[20 \mu \mathrm{g} / \mathrm{litre}, 3.5 \mathrm{ppm}]: 2 \mathrm{M} 2 \mathrm{~F}$ rats: $15 \times 6$-hr exposures: no toxic signs: autopsy, organs normal

\section{Ethers}

Dimethoxymethane $\quad(\mathrm{MeO})_{2} \mathrm{CH}_{2}$

[methylal]

liq. b.p. $41-2^{\circ} \mathrm{C}$

$4000 \mathrm{ppm}(\mathrm{D}): 4 \mathrm{~F}$ rats: $8 \times 6-\mathrm{hr}$ exposures: no toxic signs: autopsy, organs normal

Methoxyethene

$\mathrm{MeO} \cdot \mathrm{CH}: \mathrm{CH}_{2}$

[methyl vinyl ether]

vapour b.p. $5 \cdot 5^{\circ} \mathrm{C}$ (supplied in cylinder)

2000 ppm (F): 4M 4F rats: $15 \times 6-\mathrm{hr}$ exposures: no toxic signs, blood and urine tests normal: autopsy, organs normal

Isobutoxyethene

[isobutyl vinyl ether]

$\mathrm{Me}_{2} \mathrm{CH} \cdot \mathrm{CH}_{2} \cdot \mathrm{O} \cdot \mathrm{CH}: \mathrm{CH}_{2}$

liq. b.p. $83^{\circ} \mathrm{C}$

Saturated (A): $2 \mathrm{M} 2 \mathrm{~F}$ rats: $1 \times 15$-min exposure: rapid anaesthesia, all died

1000 ppm (D): $4 \mathrm{M}$ 4F rats: $15 \times 6-\mathrm{hr}$ exposures: 
initial lachrymation, low weight gain, blood tests normal: autopsy (histol.) slight lung inflammation $500 \mathrm{ppm}$ (D): $4 \mathrm{M} \mathrm{4F}$ rats: $15 \times 6-\mathrm{hr}$ exposures: low weight gain (f), blood and urine tests normal: autopsy, organs normal

$250 \mathrm{ppm}$ (D): $4 \mathrm{M}$ 4F rats: $15 \times 6-\mathrm{hr}$ exposures: no toxic signs, blood and urine tests normal: autopsy, organs normal

Bis-2-methoxyethyl ether [diethyleneglycol dimethyl ether, diglyme]

liq. b.p. $159^{\circ} \mathrm{C}$ $\mathrm{MeO} \cdot \mathrm{CH}_{2} \cdot \mathrm{CH}_{2} \cdot \mathrm{O} \cdot \mathrm{CH}_{2} \cdot \mathrm{CH}_{2} \cdot \mathrm{OMe}$

$600 \mathrm{ppm}^{\mathrm{a}}$ (D): $4 \mathrm{M}$ 4F rats: $15 \times 6-\mathrm{hr}$ exposures: irregular weight gain, blood and urine tests normal: autopsy, thymus atrophied, adrenals congested

200 ppm $^{\mathrm{a}}$ (D): $4 \mathrm{M}$ 4F rats: $15 \times 6$-hr exposures: no toxic signs, blood and urine tests normal: autopsy, organs normal

Bis-2-ethoxyethyl ether EtO $\cdot \mathrm{CH}_{2} \cdot \mathrm{CH}_{2} \cdot \mathrm{O} \cdot \mathrm{CH}_{2} \cdot \mathrm{CH}_{2} \cdot \mathrm{OEt}$ [diethylene glycol diethyl ether, diethyl carbitol]

liq. b.p. $188^{\circ} \mathrm{C}$

Saturated (A) $[2.6 \mathrm{mg} /$ litre $400 \mathrm{ppm}]: 4 \mathrm{M}$ rats: $17 \times 7$-hr exposures: restlessness: autopsy, organs normal

Bis-2-chloro-1-methylethylether liq. b.p. $187^{\circ} \mathrm{C}$

$700 \mathrm{ppm}(\mathrm{D}): 2 \mathrm{M}$ 2F rats: $1 \times 5$-hr exposure: nose and eye irritation, respiratory difficulty, 2 died later: autopsy (histol.) congestion of liver and kidneys

$350 \mathrm{ppm}$ (D): $4 \mathrm{M}$ 4F rats: $8 \times 5-\mathrm{hr}$ exposures: lethargy, respiratory difficulty, retarded weight gain: autopsy (histol.) congestion of liver and kidneys

$70 \mathrm{ppm}$ (D): $4 \mathrm{M}$ 4F rats: $20 \times 6-\mathrm{hr}$ exposures: lethargy, weight gain retarded: autopsy, organs normal

$20 \mathrm{ppm}$ (D, ethanol): $4 \mathrm{M} 4 \mathrm{~F}$ rats: $20 \times 6-\mathrm{hr}$ exposures: no toxic signs: autopsy, organs normal

\section{Aldehydes and ketones}

Propionaldehyde

[propanal]

liq. b.p. $46-50^{\circ} \mathrm{C}$

Saturated (A): $2 \mathrm{M} 2 \mathrm{~F}$ rats: $1 \times 30$-min exposure: anaesthetized, all died

$1300 \mathrm{ppm}$ (D): $4 \mathrm{M}$ 4F rats: $6 \times 6$-hr exposures: no weight gain: autopsy (histol.) liver cell vacuolation

$90 \mathrm{ppm}$ (D): $4 \mathrm{M}$ 4F rats: $20 \times 6-\mathrm{hr}$ exposures: no toxic signs: autopsy, organs normal

n-Butyraldehyde

[butanal]

liq. b.p. $75^{\circ} \mathrm{C}$

$1000 \mathrm{ppm}$ (D): $3 \mathrm{M} \mathrm{4F}$ rats: $12 \times 6-\mathrm{hr}$ exposures: no toxic signs: autopsy, organs normal
Isobutyraldehyde (92\%)

$\mathrm{Me}_{2} \mathrm{CH} \cdot \mathrm{CHO}$

[isobutanal]

liq. b.p. $64^{\circ} \mathrm{C}$

1000 ppm (D): $4 \mathrm{M}$ 4F 1ats: $12 \times 6-\mathrm{hr}$ exposures: slight nose irritation: autopsy, organs normal

\section{5-Bromopentan-2-one} liq. b.p. $80^{\circ} \mathrm{C}(13 \mathrm{~mm})$

$\mathrm{BrCH}_{2} \cdot \mathrm{CH}_{2} \cdot \mathrm{CH}_{2} \cdot \mathrm{CO} \cdot \mathrm{CH}_{3}$

Saturated (A): $3 \mathrm{M}$ rats: $9 \times 7$-hr exposures: eye irritation, salivation, slight narcosis, respiratory difficulty, slight convulsions, rectal temp. $<35^{\circ} \mathrm{C}$ : autopsy, organs congested, lungs haemorrhagic, (histol.) thickening of alveolar walls

Mist (?) $19 \mathrm{ppm}$ [D]: $7 \mathrm{M}$ rats: $15 \times 6-\mathrm{hr}$ exposures: slight lethargy, no organ damage

Acids

Acrylic acid

[propenoic acid]

liq. b.p. $142^{\circ} \mathrm{C}$

Saturated (A) [19 mg/litre, $6000 \mathrm{ppm}]$ : $2 \mathrm{M}$ 2F rats: $1 \times 5-h r$ exposure: nose and eye irritation, respiratory difficulty, unresponsive, 1 died: autopsy (histol.) lung haemorrhage, liver and kidney tubulesdegenerative changes

$1500 \mathrm{ppm}$ (D) 4M 4F rats: $4 \times 6$-hr exposures: nasal discharge, lethargy, weight loss: autopsy (histol.) kidneys congested

$300 \mathrm{ppm}(\mathrm{D}): 4 \mathrm{M} 4 \mathrm{~F}$ rats: $20 \times 6$-hr exposures: some nose irritation, lethargy, retarded weight gain: autopsy, organs normal

$80 \mathrm{ppm}(\mathrm{D}): 4 \mathrm{M}$ 4F rats: $20 \times 6-\mathrm{hr}$ exposures: no toxic signs: autopsy, organs normal

Methacrylic acid

[2-methylpropenoic acid]

liq. b.p. $161^{\circ} \mathrm{C}$

Saturated $[4.5 \mathrm{mg} / \mathrm{litre}, 1300 \mathrm{ppm}]: 2 \mathrm{M} 2 \mathrm{~F}$ rats: $5 \times 5-\mathrm{hr}$ exposures: nose and eye irritation, weight loss, blood and urine tests normal: autopsy, organs normal

$300 \mathrm{ppm}$ (D): $4 \mathrm{M}$ 4F rats: $20 \times 6$-hr exposures: no toxic signs: autopsy, organs normal (slight renal congestion?)

\section{Adipic acid}

[hexanedioic acid]

solid, m.p. $151^{\circ} \mathrm{C}$

Dust $126 \mu \mathrm{g} /$ litre (G): $2 \mathrm{M}$ 2F rats: $15 \times 6-\mathrm{hr}$ exposures: no toxic signs, blood tests normal: autopsy, organs normal

\section{Chlorinated hydrocarbons}

2-Chloropropane

[isopropyl chloride]

$\mathrm{CH}_{3} \cdot \mathrm{CHCl} \cdot \mathrm{CH}_{3}$

liq. b.p. $36 \cdot 5^{\circ} \mathrm{C}$

$1000 \mathrm{ppm}$ (D): $4 \mathrm{M}$ 4F rats: $20 \times 6-\mathrm{hr}$ exposures: 
no toxic signs: autopsy (histol.) liver-extensive vacuolation and necrosis

$250 \mathrm{ppm}$ (D): $4 \mathrm{M} \mathrm{4F}$ rats: $20 \times 6-\mathrm{hr}$ exposures: no toxic signs: autopsy, organs normal

1,1-Dichloroethene

[vinylidene chloride]

liq. b.p. $31 \cdot 9^{\circ} \mathrm{C}$

$500 \mathrm{ppm}(\mathrm{D}): 4 \mathrm{~m} 4 \mathrm{~F}$ rats: $20 \times 6$-hr exposures: nose irritation, retarded weight gain: autopsy (histol.) liver cell degeneration

$200 \mathrm{ppm}$ (D): $4 \mathrm{M}$ 4F rats: $20 \times 6$-hr exposures: slight nose irritation: autopsy, organs normal

Dichlorobutenes (mixed isomers)

1. $\mathrm{CH}_{2}: \mathrm{CH} \cdot \mathrm{CHCl} \cdot \mathrm{CH}_{2} \mathrm{Cl}$

2 and 3. cis and trans $-\mathrm{CH}_{2} \mathrm{Cl} \cdot \mathrm{CH}: \mathrm{CH} \cdot \mathrm{CH}_{2} \mathrm{Cl}$

[1. $37 \cdot 3 \%$ 3,4-dichlorobut-1-ene 2 . $17 \%$ cis-1,4dichlorobut-2-ene 3. $45 \cdot 7 \%$ trans-1,4-dichlorobut-2-ene] liq. b.p. $123-158^{\circ} \mathrm{C}$

$18 \mathrm{ppm}$ (D, pet. ether): $4 \mathrm{M} 4 \mathrm{~F}$ rats: $8 \times 6-\mathrm{hr}$ exposures: initial lachrymation, lethargy, respiratory difficulty, low rectal temp., progressive weight loss, blood and urine tests normal: autopsy, emaciated, lungs haemorrhagic, thymus atrophied, (histol.) lungs emphysematous with areas of haemorrhage and oedema

$6 \mathrm{ppm}(\mathrm{D}$, pet. ether): $4 \mathrm{M}$ 4F rats: $15 \times 6-\mathrm{hr}$ exposures: initial weight loss and lethargy, later normal: autopsy, thymus slight atrophy

2-3 ppm (D, pet. ether): $4 \mathrm{M} 4 \mathrm{~F}$ rats: $15 \times 6-\mathrm{hr}$ exposures: no toxic signs: autopsy, organs normal

Hexachlorobutadiene

liq.

$\mathrm{Cl}_{2} \mathrm{C}: \mathrm{CCl} \cdot \mathrm{CCl}: \mathrm{CCl}_{2}$

$250 \mathrm{ppm}$ (D): $4 \mathrm{M} \mathrm{4F}$ rats: $2 \times 4$-hr exposures: eye and nose irritation, respiratory difficulty, females affected more than males, apparent recovery after exposure: autopsy (histol.) degeneration of middle renal proximal tubules and of adrenal cortex

$100 \mathrm{ppm}$ (D): $4 \mathrm{M}$ 4F rats: $12 \times 6-\mathrm{hr}$ exposures: eye and nose irritation, respiratory difficulty, poor condition, weight loss, slight anaemia in females, urine tests normal, 2 females died: autopsy, kidneys pale and enlarged, adrenals enlarged, degeneration of renal cortical tubules with epithelial regeneration

$25 \mathrm{ppm}$ (D, pet. ether): 4M 4F rats: $15 \times 6$-hr exposures: poor condition, diminished weight gain in females, respiratory difficulty, blood and urine tests normal: autopsy, kidneys pale and enlarged, (histol.) damage to renal proximal tubules

$10 \mathrm{ppm}(\mathrm{D}$, pet. ether): $4 \mathrm{M}$ 4F rats: $15 \times 6-\mathrm{hr}$ exposures: retarded weight gain in females: autopsy, organs normal

$5 \mathrm{ppm}(\mathrm{D}$, pet. ether): $4 \mathrm{M} 4 \mathrm{~F}$ rats: $15 \times 6-\mathrm{hr}$ exposures: no toxic signs: autopsy, organs normal

(2-Bromoethoxy)benzene

[2-phenoxyethyl bromide]

solid, m.p. $30^{\circ} \mathrm{C}$, b.p. ca $145^{\circ} \mathrm{C}(50 \mathrm{~mm})$

Saturated $\left(\mathrm{A}, 37^{\circ} \mathrm{C}\right)[0.4 \mathrm{mg} / \mathrm{litre}, 80 \mathrm{ppm}]: 3 \mathrm{~F}$ rats: $13 \times 5 \cdot 5-\mathrm{hr}$ exposures: discomfort, lethargy, initial weight loss: autopsy, organs normal
1,2,4-Trichlorobenzene (up to $20 \%$ 1,2,3-trichlorobenzene)

liq. b.p. $206-225^{\circ} \mathrm{C}$

$\mathrm{C}_{6} \mathrm{H}_{3} \mathrm{Cl}_{3}$

Saturated (A) $[2.5 \mathrm{mg} /$ litre, $200 \mathrm{ppm}]: 2 \mathrm{M} 2 \mathrm{~F}$ rats: $15 \times 6$-hr exposures: lethargy, retarded weight gain: autopsy, organs normal

$70 \mathrm{ppm}(\mathrm{D}): 2 \mathrm{M}$ 2F rats: $15 \times 6$-hr exposures: initial lachrymation, lethargy, retarded weight gain: autopsy, organs normal

20 ppm (D, ethanol): $4 \mathrm{M}$ 4F rats: $20 \times 6-\mathrm{hr}$ exposures: no toxic signs: autopsy, organs normal

Hexafluorobenzene

liq. b.p. $80^{\circ} \mathrm{C}$

$1000 \mathrm{ppm}$ (D): $4 \mathrm{M} 4 \mathrm{~F}$ rats: $6 \times 6$-hr exposures: no weight gain (F): autopsy (histol.) lungs-macrophages, spleen-reactive hyperplasia

$500 \mathrm{ppm}$ (D): $4 \mathrm{M} 4 \mathrm{~F}$ rats: $15 \times 6-\mathrm{hr}$ exposures: no porphyrinuria, blood and urine tests normal, weight gain retarded $(F)$ : autopsy, organs normal

$250 \mathrm{ppm}$ (D): $4 \mathrm{M}$ 4F rats: $15 \times 6-\mathrm{hr}$ exposures: no toxic signs: autopsy, organs normal

Chloropentafluorobenzene

solid, m.p. $18 \cdot 1^{\circ} \mathrm{C}$, b.p. $116^{\circ} \mathrm{C}$

$1000 \mathrm{ppm}$ (D): $4 \mathrm{M}$ 4F rats: $4 \times 6-\mathrm{hr}$ exposures: lethargy, incoordination, no porphyrinuia: autopsy, organs normal

$500 \mathrm{ppm}$ (D): $4 \mathrm{M}$ 4F rats: $15 \times 6$-hr exposures: unresponsive, no porphyrinuria: autopsy, organs normal

$50 \mathrm{ppm}$ (D): $4 \mathrm{M}$ 4F rats: $15 \times 6-\mathrm{hr}$ exposures: no toxic signs: autopsy, organs normal

1,3-Dichlorotetrafluorobenzene (contains about $5 \%$ 1,2-isomer)

liq. b.p. $156^{\circ} \mathrm{C}$

Saturated (A) [30 mg/litre, $3000 \mathrm{ppm}]: 2 \mathrm{M} 2 \mathrm{~F}$ rats: $1 \times 30$-min exposure: eye irritation, nasal discharge, respiratory difficulty, light narcosis

1000 ppm (D): 4M 4F rats: $4 \times 6$-hr exposures: light narcosis with recovery overnight, increased urinary coproporphyrin (M), porphobilinogen ( $M$ and $F)$ : autopsy (histol.) damage to kidney tubules

500 ppm (D): $4 \mathrm{M} 4 \mathrm{~F}$ rats: $15 \times 6-\mathrm{hr}$ exposures: light narcosis with recovery overnight: weight gain retarded, blood and urine tests normal: autopsy (histol.) slight kidney tubular lesions

$100 \mathrm{ppm}$ (D): $4 \mathrm{M}$ 4F rats: $15 \times 6-\mathrm{hr}$ exposures: no toxic signs: autopsy, organs normal

1,3,5-Trichlorotrifluorobenzene $\quad \mathrm{C}_{6} \mathrm{~F}_{3}-1,3,5-\mathrm{Cl}_{3}$ solid, m.p. $50-60^{\circ} \mathrm{C}$

Saturated (B) $[3.6 \mathrm{mg} /$ litre, $380 \mathrm{ppm}]: 2 \mathrm{M}$ 2F rats: $2 \times 6-\mathrm{hr}$ exposures: nose irritation, respiratory difficulty, narcosis, males died, increased urinary protein and porphobilinogen, high blood urea: autopsy (histol.) liver-focal necrosis and centrilobular vacuolation with fatty changes, kidneytubular necrosis

$83 \mathrm{ppm}(\mathrm{C}): 4 \mathrm{M} 4 \mathrm{~F}$ rats: $15 \times 6$-hr exposures: no toxic signs, blood and urine tests normal: autopsy, livers enlarged 
1-Chloronaphthalene (technical)

liq. b.p. $250-280^{\circ} \mathrm{C}$

Saturated (A) $[0.25 \mathrm{mg} / \mathrm{litre}, 37 \mathrm{ppm}]: 3 \mathrm{~F}$ rats: $15 \times 6-\mathrm{hr}$ exposures: no toxic signs: autopsy, organs normal

Esters

Vinyl acetate

liq. b.p. $72^{\circ} \mathrm{C}$

Saturated (A): 4M 4F rats: $5 \mathrm{~min}$ : rapid anaesthesia, all died

$2000 \mathrm{ppm}$ (D): $4 \mathrm{M} \mathrm{4F}$ rats: $15 \times 6$-hr exposures: eye and nose irritation, respiratory difficulty, poor condition, low weight gain: autopsy (histol.) excess macrophages in lungs

$630 \mathrm{ppm}$ (D): $4 \mathrm{M}$ 4F rats: $15 \times 6$-hr exposures: low weight gain (F): autopsy, organs normal

$250 \mathrm{ppm}$ (D): $4 \mathrm{M}$ 4F rats: $15 \times 6$-hr exposures: low weight gain (F), blood and urine tests normal: autopsy, organs normal

$100 \mathrm{ppm}$ (D): $4 \mathrm{M}$ 4F rats: $15 \times 6$-hr exposures: no toxic signs: autopsy, organs normal

Methyl salicylate

[methyl 2-hydroxybenzoate]

$\mathrm{C}_{6} \mathrm{H}_{4}(\mathrm{OH})-2-\mathrm{CO}_{2} \mathrm{Me}$

liq. b.p. $223^{\circ} \mathrm{C}$

Saturated (A) $\left[700 \mathrm{mg} / \mathrm{m}_{3}, 120 \mathrm{ppm}\right]: 4 \mathrm{~F}$ rats: $20 \times 7-$ hr exposures: no toxic signs: autopsy, organs normal

Methyl isothiocyanate

liq.

$\mathrm{CH}_{3} \cdot \mathrm{NCO}$

$10 \mathrm{ppm}(\mathrm{D}$, pet. ether): $4 \mathrm{M}$ 4F rats: $15 \times 6-\mathrm{hr}$ exposures: lethargy, low weight gain (F), blood and urine tests normal: autopsy, thymus small, (histol.) organs normal

$2.5 \mathrm{ppm}$ (D, pet. ether): $4 \mathrm{M}$ 4F rats: $15 \times 6-\mathrm{hr}$ exposures: no toxic signs: autopsy, organs normal

2-Ethylhexyl acrylate

[2-ethylhexyl propenoate]

liq. b.p. $215-219^{\circ} \mathrm{C}$

$\mathrm{CH}_{2}: \mathrm{CH} \cdot \mathrm{CO} \cdot \mathrm{O} \cdot \mathrm{CH}_{2} \cdot \mathrm{CHEt} \cdot\left[\mathrm{CH}_{2}\right]_{3} \cdot \mathrm{CH}_{3}$

Saturated (A) [1 mg/litre, $130 \mathrm{ppm}]: 2 \mathrm{M}$ 2F rats: $13 \times 6$-hr exposures: initial weight loss, lethargy, slight respiratory difficulty, blood and urine tests normal: autopsy, organs normal

$50 \mathrm{ppm}$ (D, ethanol): $2 \mathrm{M} 2 \mathrm{~F}$ rats: no toxic signs: autopsy, organs normal

2-Ethylhexyl methacrylate

[2-ethylhexyl 2-methylpropenoate]

liq. b.p. $224^{\circ} \mathrm{C}$

$\mathrm{CH}_{3}: \mathrm{CMe} \cdot \mathrm{CO} \cdot \mathrm{O} \cdot \mathrm{CH}_{2} \cdot \mathrm{CHEt} \cdot\left[\mathrm{CH}_{2}\right]_{3} \cdot \mathrm{CH}_{3}$

Saturated (A) $[0.15 \mathrm{mg} / \mathrm{litre}, 60 \mathrm{ppm}], 4 \mathrm{M}$ 4F rats: $15 \times 6$-hr exposures: no toxic signs, blood and urine tests normal: autopsy (histol.) increased cellularity in lungs

$25 \mathrm{ppm}$ (D, ethanol): 4M 4F rats: $15 \times 6$-hr exposures: no toxic signs: autopsy, organs normal
Octyl methacrylate

[octyl 2-methylpropenoate]

liq. b.p. $112^{\circ} \mathrm{C}(10 \mathrm{~mm})$

Saturated (A): $2 \mathrm{M} 2 \mathrm{~F}$ rats: $20 \times 6$-hr exposures: no toxic signs: autopsy, organs normal

Lauryl methacrylate

$\mathrm{CH}_{2}: \mathrm{CMe} \cdot \mathrm{CO} \cdot \mathrm{O} \cdot \mathrm{C}_{22} \mathrm{H}_{25}$

[dodecyl 2-methylpropenoate]

(contains $25 \% \mathrm{C}_{14}$ ester)

liq. b.p. $170^{\circ} \mathrm{C}(10 \mathrm{~mm}), 205^{\circ} \mathrm{C}(50 \mathrm{~mm})$

Saturated (A): $2 \mathrm{M} 2 \mathrm{~F}$ rats: $20 \times 6-\mathrm{hr}$ exposures: no toxic signs: autopsy, organs normal

Cetostearyl methacrylate

[cetyl to stearyl 2-methylpropenoate] $\mathrm{CH}_{2}$ :CMe.CO.OR [R $=\mathrm{C}_{18} \mathrm{H}_{32}$ to $\mathrm{C}_{18} \mathrm{H}_{37}$ ] solid, m.p. $20^{\circ} \mathrm{C}$

Saturated (B): $2 \mathrm{M}$ 2F rats: $15 \times 6-\mathrm{hr}$ exposures: no toxic signs: autopsy, organs normal

2-Dimethylaminoethyl methacrylate

[2-dimethylaminoethyl 2-methylpropenoate]

liq. b.p. $187^{\circ} \mathrm{C}$

$\mathrm{CH}_{2}: \mathrm{CMe} \cdot \mathrm{CO} \cdot \mathrm{O} \cdot \mathrm{CH}_{2} \cdot \mathrm{CH}_{2} \cdot \mathrm{NMe}_{2}$

Mist (?) $250 \mathrm{ppm}$ (D): $4 \mathrm{M}$ 4F rats: $15 \times 6$-hr exposures: nose and eye irritation, rapid breathing, weight gain low and irregular, blood and urine tests normal: autopsy, organs normal

$100 \mathrm{ppm}$ (D): $4 \mathrm{M} 4 \mathrm{~F}$ rats: $15 \times 6-\mathrm{hr}$ exposures: no toxic signs: autopsy, organs normal

2-Hydroxyethyl methacrylate

[2-hydroxyethyl 2-methylpropenoate]

liq. $\mathrm{CH}_{2}: \mathrm{CMe} \cdot \mathrm{CO} \cdot \mathrm{O} \cdot \mathrm{CH}_{2} \cdot \mathrm{CH}_{2} \mathrm{OH}$

Saturated (A) $[0.5 \mathrm{mg} / \mathrm{litre}, 90 \mathrm{ppm}]: 4 \mathrm{M}$ 4F rats: $15 \times 6$-hr exposures: erratic weight gain (F): autopsy, organs normal

2-Hydroxypropyl methacrylate

[2-hydroxypropyl 2-methylpı openoate $80 \%$ ]

$\mathrm{CH}_{2}: \mathrm{CMe} \cdot \mathrm{CO} \cdot \mathrm{O} \cdot \mathrm{CH}_{2} \cdot \mathrm{CH}(\mathrm{OH}) \cdot \mathrm{CH}_{3}$

[2-hydroxy-1-methylethyl 2-methylpropenoate $20 \%$ ]

liq. b.p. $79^{\circ} \mathrm{C}(5 \mathrm{~mm})$

$\mathrm{CH}_{2}: \mathrm{CMe} \cdot \mathrm{CO} \cdot \mathrm{O} \cdot \mathrm{CHMe} \cdot \mathrm{CH}_{2} \mathrm{OH}$

Saturated (A) $[0.5 \mathrm{mg} /$ litre, $90 \mathrm{ppm}]: 4 \mathrm{M} 4 \mathrm{~F}$ rats: $15 \times 6$-hr exposures: no toxic signs: autopsy, organs normal

Glycol dimethacrylate

[ethylene bis-(2-methylpropenoate)]

liq. b.p. $120^{\circ} \mathrm{C}(20 \mathrm{~mm})$

Saturated (A) [1 mg/litre, $120 \mathrm{ppm}]$ : $3 \mathrm{~F}$ rats: $13 \times 6-\mathrm{hr}$ exposures: slight lethargy: autopsy (histol.) alveolar walls thickened, peribronchiolar lymphocytic reaction

Methyl chloroformate

[methyl chloromethanoate]

$\mathrm{Cl} \cdot \mathrm{CO} \cdot \mathrm{OMe}$

liq. b.p. $72^{\circ} \mathrm{C}$

$20 \mathrm{ppm}$ (D, pet. ether): 4M 4F rats: $15 \times 6-\mathrm{hr}$ exposures: nose i1 ritation, respiratory difficulty, lethargy, poor condition, weight loss: autopsy, lungs distended and haemorrhagic, (histol.) areas of consolidation 
and collapse and some oedema and haemorihage in lungs, kidneys congested

5 ppm (D, pet. ether): $4 \mathrm{M}$ 4F rats: $15 \times 6-\mathrm{hr}$ exposures: nose irritation, lethargy: autopsy, organs normal

$1 \mathrm{ppm}(\mathrm{D}$, pet. ether): $4 \mathrm{M}$ 4F rats: $15 \times 6$-hr exposures: no toxic signs: autopsy, organs normal

Ethyl chloroformate

[ethyl chloromethanoate]

liq. b.p. $95^{\circ} \mathrm{C}$

20 ppm (D, pet. ether): $4 \mathrm{M} \mathrm{4F}$ rats: $10 \times 6-\mathrm{hr}$ exposures: nose irritation, respiratory difficulty, poor condition, weight loss: autopsy, lungs distended, (histol.) lung haemorrhage

5 ppm (D, pet. ether): $4 M$ 4F rats: $20 \times 6$-hr exposures: retarded weight increase: autopsy, organs normal

$1 \mathrm{ppm}(\mathrm{D}$, pet. ether): $4 \mathrm{M} 4 \mathrm{~F}$ rats: $20 \times 6-\mathrm{hr}$ exposures: no toxic signs: autopsy, organs normal

Isopropyl chloroformate

[isopropyl chloromethanoate]

liq. $103-105^{\circ} \mathrm{C}$ (decomp.)

$200 \mathrm{ppm}(\mathrm{D}): 4 \mathrm{M}$ 4F rats: $1 \times 5$-hr exposure: $2 \mathrm{M}$ rats: dyspnoea, both died later

$50 \mathrm{ppm}$ (D): $4 \mathrm{M} 4 \mathrm{~F}$ rats: $11 \times 6-\mathrm{hr}$ exposures: Iespiratory difficulty, weight loss, 1 died: autopsy (histol.) lung haemorrhage

$20 \mathrm{ppm}$ (D, isopropanol): $4 \mathrm{M}$ 4F rats: $20 \times 6-\mathrm{hr}$ exposures: nasal irritation: autopsy, organs normal

5 ppm (D, isopropanol): $4 \mathrm{M} 4 \mathrm{~F}$ rats: no toxic signs: autopsy, organs normal

Methyl nitrite

vapour (b.p. $-12^{\circ} \mathrm{C}$ )

250 ppm $^{\mathrm{e}}$ (I): $4 \mathrm{M} 4 \mathrm{~F}$ rats: $1 \times 4$-hr exposure: rats gasping and pale, 7 died

110 ppm $^{\mathrm{e}}$ (I): $4 \mathrm{M}$ 4F rats: $13 \times 6$-hr exposures: good condition though pale, methaemoglobin rose to $30-40 \%$ of total $\mathrm{Hb}$ by end of each exposure with recovery overnight: autopsy, organs normal

84 ppme $^{\mathrm{e}}(\mathrm{I}): 4 \mathrm{M} \mathrm{4F}$ rats: $15 \times 6-\mathrm{hr}$ exposures: good condition, methaemoglobin 10\%: autopsy, organs normal

25 ppm $^{\mathrm{e}}$ (I): $4 \mathrm{M}$ 4F rats: $15 \times 6-\mathrm{hr}$ exposures: no toxic signs: autopsy, organs normal

$35 \mathrm{ppm}^{\mathrm{e}}$ (I): $1 \mathrm{~F}$ cat $(3.4 \mathrm{~kg}): 1 \times 6-\mathrm{hr}$ exposure: behaviour normal, methaemoglobin $6 \%$

Dimethyl carbonate $\quad \mathrm{Me}_{2} \mathrm{CO}_{3}$

liq. b.p. $90 \cdot 2^{\circ} \mathrm{C}$

Saturated (A) [5000 ppm, $20 \mathrm{mg} /$ litre]: $2 \mathrm{M} 2 \mathrm{~F}$ rats: $1 \times 6-h r$ exposure: eye irritation, salivation, respiratory difficulty, incoordination, rapid recovery after exposure: autopsy, organs normal

$1000 \mathrm{ppm}(\mathrm{D}): 2 \mathrm{M} 2 \mathrm{~F}$ rates: $15 \times 6-\mathrm{hr}$ exposures: no toxic signs: autopsy, organs normal

\section{Amino compounds}

s-Butylamine

[2-aminobutane]

liq. b.p. $62^{\circ} \mathrm{C}$
Saturated (A) $[280 \mathrm{mg} / \mathrm{litre}, 10 \% \mathrm{v} / \mathrm{v}]: 7 \mathrm{M}$ rats: $1 \times 5$-hr exposure: intense eye and nose irritation respiratory difficulty, convulsions, all died: autopsy, cornea opaque and white, (histol.) organs normal

$233 \mathrm{ppm}(\mathrm{D}): 7 \mathrm{M}$ rats: $13 \times 6 \cdot 5-\mathrm{hr}$ exposures: discomfort, lethargy, retarded weight gain: autopsy, organs normal

Di-s-butylamine

[bis-(2-methylpropyl)amine]

$\left(\mathrm{CH}_{3} \cdot \mathrm{CH}_{2} \cdot \mathrm{CHMe}\right)_{2} \mathrm{NH}$

liq. b.p. $135^{\circ} \mathrm{C}$

Saturated (A) $[50 \mathrm{mg} /$ litre, $1 \% \mathrm{v} / \mathrm{v}]: 4 \mathrm{M} 4 \mathrm{~F}$ rats: $19 \times 6 \cdot 5-\mathrm{hr}$ exposures: restlessness, initial tremors, incoordination, no weight gain: autopsy, organs normal

Tributylamine

$\mathrm{Bu}_{3} \mathrm{~N}$

liq.

$120 \mathrm{ppm}$ (D): $4 \mathrm{M}$ 4F rats: $19 \times 6-\mathrm{hr}$ exposures: nose irritation, restlessness, incoordination and tremors, no weight gain: autopsy, organs normal

$62 \mathrm{ppm}(\mathrm{D}$, pet, ether): $4 \mathrm{M}$ 4F rats: $19 \times 6-\mathrm{hr}$ exposures: lethargy, no weight gain: autopsy, organs normal

$29 \mathrm{ppm}$ (D, pet. ether): 4M 4F rats: $19 \times 6-\mathrm{hr}$ exposures: slight lethargy: autopsy, organs normal

Nonylamine $\quad \mathrm{CMe}_{3} \cdot \mathrm{CH}_{2} \cdot \mathrm{CHMe} \cdot\left(\mathrm{CH}_{2}\right)_{2} \cdot \mathrm{NH}_{2}$

[1-amino-3,5,5-trimethylhexane]

liq. b.p. $176^{\circ} \mathrm{C}$

Saturated (A) [2 mg/litre, $340 \mathrm{ppm}]: 3 \mathrm{~F}$ rats: $1 \times 35-$ min exposure: nose and eye irritation, salivation, tremors: autopsy, organs normal

$66 \mathrm{ppm}(\mathrm{D}): 6 \mathrm{~F}$ rats: $6 \times 6$-hr exposures: nose and eye irritation, tremors: autopsy, organs normal

$16.5 \mathrm{ppm}$ (D, pet. ether): $6 \mathrm{~F}$ rats: $10 \times 6$-hr exposures: no toxic signs: al'topsy, organs normal

Dinonylamine

[bis-(3,5,5-trimethylhexyl)amine]

liq. b.p. $172^{\circ} \mathrm{C}(20 \mathrm{~mm})$

Saturated (A) $[0.16 \mathrm{mg} /$ litre, $15 \mathrm{ppm}]: 3 \mathrm{~F}$ rats: $14 \times 6$-hr exposures: slight restlessness: autopsy, organs normal

Trinonylamine

[tris-(3,5,5-trimethylhexyl)amine]

liq. b.p. $216^{\circ} \mathrm{C}(20 \mathrm{~mm})$

Saturated $(0.08 \mathrm{mg} / \mathrm{litre}, 7 \mathrm{ppm}): 3 \mathrm{~F}$ rats: $8 \times 6-\mathrm{hr}$ exposures: no toxic signs: autopsy, no organ damage

1,6-Diaminohexane

[hexamethylenediamine]

$\mathrm{NH}_{2}\left(\mathrm{CH}_{2}\right)_{6} \mathrm{NH}_{2}$

liq. b.p. $200^{\circ} \mathrm{C}$ (supplied as $90 \%$ aqueous solution)

Fume (H) $10 \mathrm{mg} /$ litre: $4 \mathrm{M}$ 4F rats: $2 \times 6-\mathrm{hr}$ exposures: nose irritation, respiratory difficulty, lethargy, 1M 1F died: autopsy, lungs congested, (histol.) peribronchiolar inflammation, areas of haemorrhage and oedema in lungs, vacuolation of kidney tubules

Fume (H) $5 \mathrm{mg}$ /litre: $4 \mathrm{M} 4 \mathrm{~F}$ rats: $11 \times 6$-hr exposures: nose and lung irritation, little weight gain, lethargy, poor condition, 1 died, urine and blood tests normal: autopsy, petechial haemorrhage in lungs, (histol.) lung inflammation 
Fume $(\mathrm{H}) 1 \mathrm{mg}$ /litre: 4M 4F rats: $15 \times 6$-hr exposures: no toxic signs, (histol.) organs normal

Diethylenetriamine

[bis-2-aminoethylamine]

liq. b.p. $208^{\circ} \mathrm{C}$

Saturated (A) $[0.55 \mathrm{mg} /$ litre, $130 \mathrm{ppm}]: 2 \mathrm{M} 2 \mathrm{~F}$ rats: $15 \times 6$-hr exposures: no toxic signs (hair coarsened): autopsy, organs normal

2-Aminobutan-1-ol

liq. b.p. $178^{\circ} \mathrm{C}$

Saturated (A) $[0.3 \mathrm{mg} / \mathrm{litre}, 85 \mathrm{ppm}]: 2 \mathrm{M} 2 \mathrm{~F}$ rats: $15 \times 6-\mathrm{hr}$ exposures: increased white cell count, high blood urea, urine tests normal: autopsy, organs normal

$50 \mathrm{ppm}(\mathrm{D}): 4 \mathrm{M} 4 \mathrm{~F}$ rats: $15 \times 6$-hr exposures: no toxic signs, blood and urine tests normal: autopsy, organs normal

1-Diethylaminopentan-2-one $\mathrm{CH}_{3} \cdot\left(\mathrm{CH}_{2}\right)_{2} \cdot \mathrm{CO} \cdot \mathrm{CH}_{2} \cdot \mathrm{NEt}_{2}$ liq. b.p. $100^{\circ}(23 \mathrm{~mm})$

Saturated (A): $3 \mathrm{M}$ rats: $10 \times 6$-hr exposures: eye irritation, salivation, no weight gain: autopsy (histol.) slight thickening of alveolar walls

$78 \mathrm{ppm}$ (D): $7 \mathrm{M}$ rats: $14 \times 6-\mathrm{hr}$ exposures: slight nasal irritation: autopsy (histol.) slight thickening of alveolar walls

\section{Nitriles}

n-Propyl cyanide

[1-cyanopropane]

liq. b.p. $116-7^{\circ} \mathrm{C}$

Saturated (A) [ca $2 \% \mathrm{v} / \mathrm{v}]: 4 \mathrm{M}$ rats: $1 \times 1-\mathrm{hr}$ exposure: eye and nose irritation, respiratory difficulty, coma, all died

$2000 \mathrm{ppm}$ (D): $4 \mathrm{M}$ 4F rats: $1 \times 4$-hr exposure: respiratory difficulty, weight loss, lethargy, convulsions, rectal temp. $<32^{\circ} \mathrm{C}$ : autopsy, lungs congested

$400 \mathrm{ppm}$ (D): $4 \mathrm{M}$ 4F rats: $2 \times 5 \cdot 5-\mathrm{hr}$ exposures: lethargy, weight loss, low body temperature, gasping, 1 died: autopsy, organs normal

$200 \mathrm{ppm}$ (D): $4 \mathrm{M}$ 4F rats: $20 \times 6-\mathrm{hr}$ exposures: no toxic signs, daily urinary thiocyanate $6 \mu \mathrm{g}$ rat (normal 0.07 $\mu \mathrm{g}$ ): autopsy, organs normal

Chloroacetonitrile

[chloromethyl cyanide]

liq. b.p. $124^{\circ} \mathrm{C}$

Saturated (A) [34 mg/litre, 1\% v/v]: 2M 2F rats: $1 \times 30$-min exposure: acute lachrymation, all died

$300 \mathrm{ppm}$ (D): $2 \mathrm{M} 2 \mathrm{~F}$ rats: $3 \times 5-\mathrm{hr}$ exposures: respiratory difficulty, lachrymation, incoordination, low body temperature, weight loss: autopsy, organs congested

$80 \mathrm{ppm}$ (D): $4 \mathrm{M}$ 4F rats: $6 \times 6$-hr exposures: slight respiratory difficulty, lethargy, low weight gain: autopsy, lungs congested, (histol.) congestion of kidney, liver and spleen

$20 \mathrm{ppm}$ (D, isopropanol): $4 \mathrm{M}$ 4F rats: $20 \times 6-\mathrm{hr}$ exposures: no toxic signs: autopsy, slight kidney congestion

$N$-2-Cyanoethylaniline

solid, m.p. $50-51^{\circ} \mathrm{C}$

Saturated (C) $[0.07 \mathrm{mg} / \mathrm{litre}, 12 \mathrm{ppm}], 4 \mathrm{M}$ 4F rats: $15 \times 6$-hr exposures: no toxic signs: autopsy, organs normal

\section{Heterocyclics}

1-Acetyl- $\gamma$-butyrolactone

liq. b.p. $\mathrm{ca} 125^{\circ} \mathrm{C}(13 \mathrm{~mm})$

Saturated (A): $3 \mathrm{M}$ rats: $10 \times 7-\mathrm{hr}$ exposures: no toxic signs: autopsy, organs normal

2-Methyl-1,3-dioxolan

liq. b.p. $78-81^{\circ} \mathrm{C}$

$500 \mathrm{ppm}$ (D): $4 \mathrm{M}$ 4F rats: $15 \times 6-\mathrm{hr}$ exposures: retarded weight gain in females, blood and urine tests normal: autopsy, organs normal

$250 \mathrm{ppm}$ (D): $4 \mathrm{M}$ 4F rats: $15 \times 6-\mathrm{hr}$ exposures: no toxic signs: autopsy, organs normal

$N$-Formylpiperidine

$\mathrm{C}_{5} \mathrm{H}_{10} \mathrm{~N} \cdot \mathrm{CHO}$

liq. b.p. $220^{\circ} \mathrm{C}$

Saturated (A) [0.74 mg/litre, $150 \mathrm{ppm}]: 2 \mathrm{M} 2 \mathrm{~F}$ rats: $12 \times 6$-hr exposures: no toxic signs: autopsy, organs normal

2-Methylthiazole

liq. t.p. $128^{\circ} \mathrm{C}$

$100 \mathrm{ppm}(\mathrm{D}): 4 \mathrm{M} 4 \mathrm{~F}$ rats: $15 \times 6$-hir exposures: eye and nose irritation, retarded weight gain, lethargy, blood and urine tests normal: autopsy, organs normal

$35 \mathrm{ppm}(\mathrm{D}$, pet. ether): $4 \mathrm{M}$ 4F rats: $15 \times 6-\mathrm{hr}$ exposures: lethargy: autopsy, organs normal

25 ppm (D,pet. ether): 4M 4F rats: $15 \times 6$-hr exposures: no toxic signs: autopsy, organs normal

Pentachloropyridine

solid, m.p. $123-125^{\circ} \mathrm{C}$

Saturated (B) $[0.01 \mathrm{mg} /$ litre, $1 \mathrm{ppm}]: 2 \mathrm{M}$ 2F rats: $15 \times 6-h r$ exposures: no toxic signs, urine tests normal: autopsy, organs normal

2-Methylbenzoxazole

liq. b.p. $203^{\circ} \mathrm{C}$

Saturated (A) $[1.9 \mathrm{mg} /$ litre, $340 \mathrm{ppm}$ ]: $2 \mathrm{M} 2 \mathrm{~F}$ rats: $13 \times 6$-hr exposuies: eye in ritation, narcosis, weight

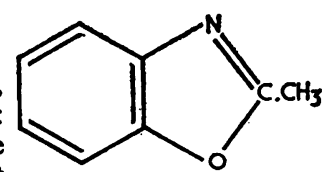
loss: autopsy, organs normal

188 ppma $^{2}$ (D): 4 M 4F rats: $15 \times 6$-hr exposures: no toxic signs, blood and urine tests normal: autopsy (histol.) slight lung inflammation

$60 \mathrm{ppm}^{\mathrm{a}}$ (D): $4 \mathrm{M} \mathrm{4F}$ rats: $15 \times 6$-hr exposures: no toxic signs: autopsy, or gans normal 
3,6-Dimethyl-1,2-benzisoxazole

liq. b.p. $70^{\circ} \mathrm{C}(0.5 \mathrm{~mm})$

Saturated (A) $[0.35 \mathrm{mg} /$ litre, $60 \mathrm{ppm}]$ : no toxic signs, blood and urine tests normal: autopsy,organs normal<smiles>Cc1noc2ccccc12</smiles>

2-Aminomethyl-3,4-dihydropyran

liq. b.p. $74^{\circ} \mathrm{C}$

$250 \mathrm{ppm}(\mathrm{D}): 4 \mathrm{M} 4 \mathrm{~F}$ rats: $1 \times 5$-hr exposure: eye and nose irritation, respiratory difficulty (M more affected), poor condition: autopsy (histol.) excess macrophages in lungs

$100 \mathrm{ppm}$ (D): 4M 4F rats: $6 \times 6$-hr exposures: nose irritation, lethargy, weight loss, blood and urine tests normal, 1M died: autopsy (histol.) excess macrophages in lungs

$10 \mathrm{ppm}(\mathrm{D}$, pet. ether): $4 \mathrm{M}$ 4F rats: $15 \times 6-\mathrm{hr}$ exposures: retarded weight gain, blood and urine tests normal: autopsy, organs normal

$5 \mathrm{ppm}$ (D, pet. ether): 4M 4F 1ats: $15 \times 6$-hr exposures: no toxic signs. autopsy, organs normal

2-Methoxy-3,4-dihydropyran liq. b.p. $127^{\circ} \mathrm{C}$

$1000 \mathrm{ppm}$ (D): $2 \mathrm{M} 2 \mathrm{~F}$ rats: $15 \times 6$-hr exposures lethargy, weight loss, blood and urine tests normal: autopsy, organs normal

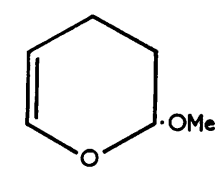

$350 \mathrm{ppm}$ (D): $4 \mathrm{M} 4 \mathrm{~F}$ rats: $15 \times 6$-hr exposures: unresponsive: autopsy, organs normal

$250 \mathrm{ppm}$ (D): $4 \mathrm{M}$ 4F rats: $15 \times 6-\mathrm{hr}$ exposures: no toxic signs: autopsy, organs normal

4-Hydroxytetrahydropyran

liq. b.p. $196^{\circ} \mathrm{C}$

Saturated (A): $6 \mathrm{~F}$ rats: $6 \times 6$-hr exposures: slight restlessness: autopsy, organs normal

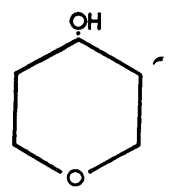

\section{3,5-Dimethylmorpholine}

liq. b.p. $143-5^{\circ} \mathrm{C}$

$750 \mathrm{ppm}(\mathrm{D}): 4 \mathrm{M}$ 4F ats: $15 \times 6$-hr exposures: nose irritation, respiratory difficulty, lethargy, weight

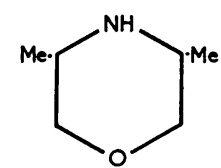

loss, urine tests normal, blood-reduced leucocyte count with increased polymorphs in females, anaemia and reticulocytosis: autopsy (histol.) hyperplasia of reticuloendothelial cells in spleen and of lymphoid tissue in lungs

$250 \mathrm{ppm}$ (D): $4 \mathrm{M} 4 \mathrm{~F}$ rats: $15 \times 6-\mathrm{hr}$ exposures: diminished weight increase $(F)$ : autopsy, organs normal

$50 \mathrm{ppm}(\mathrm{D}$, water): $4 \mathrm{M}$ 4F rats: $15 \times 6$-ht exposures: no toxic signs: autopsy, organs normal

\section{Peroxy compounds}

Ethyl t-butyl peroxyoxalate $(30.8 \% \mathrm{w} / \mathrm{w}$ in white spirit)

$\mathrm{EtO} \cdot \mathrm{CO} \cdot \mathrm{CO} \cdot \mathrm{O} \cdot \mathrm{O} \cdot \mathrm{CMe}_{3}$

Saturated (A) [1 ppm $\left.{ }^{\mathrm{b}}\right]$ : 4F rats: $15 \times 6$-hr exposures: no toxic signs: autopsy, organs normal

Cumene $\alpha$-hydroperoxide $(41.5 \% \mathrm{w} / \mathrm{w}$ in cumene)

[isopropylbenzene hydroperoxide]

Saturated (A) [50 $\left.\mathrm{ppm}^{\mathrm{c}}\right]: 2 \mathrm{~F}$ rats: $3 \times 4$-hr exposures: incoordination, tremor, narcosis, 1 died: autopsy (histol.) lungs congested, kidneys congested<smiles>O=C(O)c1ccccc1</smiles>

$31.5 \mathrm{ppm}^{\mathrm{c}}(\mathrm{D}$, ethanol): $6 \mathrm{~F}$ rats: $7 \times 5$-hr exposures: salivation, respiratory difficulty, tremors, hyperaemia of ears and tail, weight loss: autopsy (histol.) lungs-emphysema and thickening of alveolar walls

$16 \mathrm{ppm}^{\mathrm{c}}(\mathrm{D}$, ethanol): $6 \mathrm{~F}$ rats: $12 \times 4 \cdot 5$-hr exposures: salivation, nose irritation: autopsy, organs normal

Dipropionyl peroxide (22.7\% w/w in white spirit)

$\left(\mathrm{C}_{2} \mathrm{H}_{5} \cdot \mathrm{CO} \cdot \mathrm{O}\right)_{2}$

Saturated (A): $3 \mathrm{M}$ rats: $1 \times 1 \cdot 5-\mathrm{hr}$ exposure: nose and eye irritation, respiratory difficulty, all dead $1 \mathrm{hr}$ later: autopsy, lungs haemorrhagic

$100 \mathrm{ppm}^{\mathrm{b}}$ (D): $2 \mathrm{M} 2 \mathrm{~F}$ rats: $2 \times 5-\mathrm{hr}$ exposures: nose and eye irritation, respiratory difficulty, lethargy, weight loss, 1 died 2 days later: autopsy, organs normal

$30 \mathrm{ppm}^{\mathrm{b}}$ (D): $2 \mathrm{M}$ 2F rats: $4 \times 5$-hr exposures: nose irritation: autopsy, organs normal

$10 \mathrm{ppm}^{\mathrm{b}}$ (D, pet. ether): 4M 4F rats: $19 \times 5-\mathrm{hr}$ exposures: lethargy, retarded weight gain: autopsy, organs normal

$7 \mathrm{ppm}^{\mathrm{b}}$ (D, pet. ether): $4 \mathrm{M}$ 4F rats: $14 \times 5$-hr exposures: no toxic signs: autopsy, organs nor mal

t-Butyl peroxypivalate $(33 \cdot 3 \% \mathrm{w} / \mathrm{w}$ in white spirit) $\mathrm{Me}_{3} \mathrm{C} \cdot \mathrm{CO} \cdot \mathrm{O} \cdot \mathrm{O} \cdot \mathrm{CMe}_{3}$

$200 \mathrm{ppm}(\mathrm{D}): 2 \mathrm{M} 2 \mathrm{~F}$ rats: $1 \times 5$-hr exposure: nose irritation, respiratory difficulty, lethargy, weight loss: autopsy, organs normal

$50 \mathrm{ppm}(\mathrm{D}): 2 \mathrm{M}$ 2F rats: $20 \times 6-\mathrm{hr}$ exposures: no toxic signs: autopsy, organs normal

Bis-3-methylbutyl peroxydicarbonate $(20 \% \mathrm{w} / \mathrm{w}$ in white spirit) $\left(\mathrm{C}_{5} \mathrm{H}_{11} \cdot \mathrm{OCO}\right)_{2}$

Saturated (A) $\left[1.7 \mathrm{ppm}^{\mathrm{b}}\right] 2 \times 6$-hr exposures: no toxic signs, apart from slight nose inritation attributable to white spirit: autopsy, organs normal

Mist $140 \mathrm{mg} / \mathrm{m}^{3 \mathrm{~b}}$ (D, white spirit): $4 \mathrm{~F}$ rats: $3 \times 4-\mathrm{hr}$ exposures: nose and eye irritation, respiratory difficulty, weight loss: autopsy (histol.) pneumonia

Mist $44 \mathrm{mg} / \mathrm{m}^{3 \mathrm{~b}}(\mathrm{D}$, white spirit): $4 \mathrm{M}$ rats: $8 \times 5-\mathrm{hr}$ exposures: eye and nose irritation, respiratory difficulty, lethargy: autopsy (histol.) lungs-thickened alveolar walls, peribronchiolar leucocytic reaction

t-Butyl peracetate $(50 \% \mathrm{w} / \mathrm{w}$ in dimethyl phthalate) $\mathrm{CH}_{3} \cdot \mathrm{CO} \mathbf{0} \cdot \mathbf{O} \cdot \mathrm{CMe}_{3}$ 
Saturated (A) $\left[30 \mathrm{ppm}^{\mathrm{b}}\right]$ : $2 \mathrm{M}$ 2F rats: $1 \times 4-\mathrm{hr}$ exposure: nose irritation, 1espiratory difficulty: autopsy, lung oedema

$1.6 \mathrm{ppm}^{\mathrm{b}}$ (D, ethyl acetate): $2 \mathrm{M} 2 \mathrm{~F}$ rats: $8 \times 6-\mathrm{hr}$ exposures: nose irritation, respiratory difficulty, lethargy, weight loss

$0.7 \mathrm{ppm}^{\mathrm{b}}(\mathrm{D}$, ethyl acetate): $2 \mathrm{M}$ 2F rats: $20 \times 6-\mathrm{hr}$ exposures: lethargy, diminished weight increase, nose irritation

$0.15 \mathrm{ppm}^{\mathrm{b}}$ (D, ethyl acetate): $2 \mathrm{M} 2 \mathrm{~F}$ rats: $20 \times 6-\mathrm{hr}$ exposures: no toxic signs: autopsy, organs normal

\section{Organic phosphorus compounds}

Tributyl phosphite

liq. b.p. $125-127^{\circ} \mathrm{C}(15 \mathrm{~mm})$

Saturated (A) $[2 \cdot 3 \mathrm{mg} / \mathrm{litres}, 220 \mathrm{ppm}]: 2 \mathrm{M} 2 \mathrm{~F}$ rats: $15 \times 6$-hr exposures: no toxic signs, blood and urine tests normal: autopsy, organs normal

\section{1,1,1-Trishydroxymethylpropane bicyclic phosphite} solid m.p. $56^{\circ} \mathrm{C}$

Saturated (C): $4 \mathrm{M} \quad 4 \mathrm{~F}$ rats: $1 \times 1$-hr exposure: tremors, convulsions, all died during or soon after exposure: autopsy, liver, adrenals and kidneys

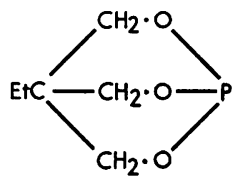

congested, lungs pale with petechial haemorrhage

$10 \mathrm{ppm}(\mathrm{D}$, pet. ether): $4 \mathrm{M}$ 4F rats: $1 \times 4-\mathrm{hr}$ exposure: tremors, convulsions, all died: autopsy, organs normal

$5 \mathrm{ppm}(\mathrm{D}$, pet. ether): $4 \mathrm{M} 4 \mathrm{~F}$ rats: $2 \times 6-\mathrm{hr}$ exposures: rapid breathing, tremors and convulsions, all died

$2.5 \mathrm{ppm}(\mathrm{D}$, pet. ether): $4 \mathrm{M} 4 \mathrm{~F}$ rats: $5 \times 6-\mathrm{hr}$ exposures: tremors on 3rd day, weight loss, 1 died: autopsy, organs normal

$1 \mathrm{ppm}$ (D, pet. ether): $4 \mathrm{M} 4 \mathrm{~F}$ rats: $15 \times 6-\mathrm{hr}$ exposures: slight transient head tremors on 4th day, retarded weight gain, blood and urine tests normal: autopsy, organs normal

$0.5 \mathrm{ppm}$ (D, pet. ether): $4 \mathrm{M} 4 \mathrm{~F}$ rats: $15 \times 6-\mathrm{hr}$ exposures: reduced weight gain (F): autopsy, organs normal

$0.25 \mathrm{ppm}$ (D, pet. ether): $4 \mathrm{M} 4 \mathrm{~F}$ rats: $15 \times 6-\mathrm{hr}$ exposures: no toxic signs: autopsy, organs normal

Phosphorus tri-isocyanate

$\mathrm{P}(\mathrm{NCO})_{3}$

liq. b.p. $168^{\circ} \mathrm{C}$

Supplied as solid polymer and depolymerized by heating at $80^{\circ} \mathrm{C}$ and $25 \mathrm{~mm}$ pressure.

Saturated (A) [6 mg/litre, $900 \mathrm{ppm}]: 2 \mathrm{M} 2 \mathrm{~F}$ rats: $1 \times 1$-hr exposure: nose and eye irritation, respiratory difficulty, autopsy, organs normal

$500 \mathrm{ppm}(\mathrm{D}$, ethyl acetate): $6 \mathrm{~F}$ rats: $2 \times 5-\mathrm{hr}$ exposures: eye and nose irritation, respiratory difficulty, weight loss: autopsy, organs normal

$O O^{\prime}$-Diethyl phosphorochloridothionate $\quad(\mathrm{EtO})_{2} \mathrm{PS} \cdot \mathrm{Cl}$ liq. b.p. $80-90^{\circ} \mathrm{C}(18 \mathrm{~mm})$

$104 \mathrm{ppm}(\mathrm{D}): 4 \mathrm{M}$ rats: $2 \times 4$-hr exposures: nose and eye irritation, salivation, respiratory difficulty, autopsy, lungs swollen, gut distended with gas, (histol.) lung emphysema

$20 \mathrm{ppm}$ (D): $4 \mathrm{M}$ rats: $14 \times 5-\mathrm{hr}$ exposures: severe nose irritation, respiratory difficulty, weight loss: autopsy (histol.) lungs-alveolar thickening and areas of collapse, degeneration of kidney tubular cortex

$5 \mathrm{ppm}(\mathrm{D}$, chloroform): $4 \mathrm{M}$ rats: $14 \times 5$-hr exposures: slight nose irritation: autopsy (histol.) lungsslight thickening of alveolar walls

\section{Silicon compounds}

Silicon tetrafluoride

compressed gas (b.p. $-95^{\circ} \mathrm{C}$ )

$1000 \mathrm{ppm}(\mathrm{E}): 4 \mathrm{~F}$ rats: $1 \times 20$-min exposure: severe nose and eye irritation, respiratory difficulty, lethargy: autopsy, organs normal

$300 \mathrm{ppm}(\mathrm{F}): 4 \mathrm{~F}$ rats: $3 \times 4 \cdot 5-\mathrm{hr}$ exposures: nose and eye irritation, respiratory difficulty, progressive deterioration of condition, 1 died: autopsy, lungs distended, (histol.) lung congestion and emphysema, liver congested, degeneration of kidney cortical tubules

$60 \mathrm{ppm}(\mathrm{F}): 4 \mathrm{~F}$ 1ats: $14 \times 6$-hr exposures: lethargy, nose irritation, weight gain retarded: autopsy, organs normal

$15 \mathrm{ppm}(\mathrm{F}): 3 \mathrm{M}$ 4F rats: $20 \times 6-\mathrm{hr}$ exposures: no toxic signs: autopsy, organs normal

Tetramethylsilane

liq. b.p. $26 \cdot 5^{\circ} \mathrm{C}$

$8000 \mathrm{ppm}(\mathrm{D}$, cooled): $4 \mathrm{M}$ rats: $1 \times 6-\mathrm{hr}$ exposure: no toxic signs: autopsy, organs normal

$1000 \mathrm{ppm}(\mathrm{D}$, cooled): $4 \mathrm{M}$ rats: $15 \times 6$-hr exposures: lethargy: autopsy, organs congested

Diphenyldimethoxysilane

liq. non-volatile $140^{\circ} \mathrm{C}(2 \mathrm{~mm})$

$\left(\mathrm{C}_{6} \mathrm{H}_{5}\right)_{2} \mathrm{Si}(\mathrm{OMe})_{2}$

Saturated (A): 4M 4F rats: $20 \times 6-\mathrm{hr}$ exposures: no toxic signs: autopsy, organs normal

Silicon tetraisocyanate

solid-liq. m.p. $26^{\circ} \mathrm{C}$, b.p. $186^{\circ} \mathrm{C}$

$\mathrm{Si}(\mathrm{NCO})_{4}$

Saturated $\left(\mathrm{A}, 30^{\circ} \mathrm{C}\right)[1.6 \mathrm{mg} / \mathrm{litre}, 200 \mathrm{ppm}]$ : $4 \mathrm{~F}$ rats: $6 \times 5$-hr exposures: eye and nose irritation, respiratory difficulty, no weight gain: autopsy, lungs and kidneys congested

$50 \mathrm{ppm}(\mathrm{D}$, ethyl acetate): $4 \mathrm{M} 4 \mathrm{~F}$ rats: $20 \times 6-\mathrm{hr}$ exposures: no toxic signs: autopsy, organs normal

\section{Sulphur compounds}

Sulphur dichloride $\mathrm{S}_{2} \mathrm{Cl}_{4}$ liq. b.p. $59^{\circ} \mathrm{C}$ [approx. $75 \%$ pure, contained $20 \% \mathrm{SCl}_{2}$ ] $340 \mathrm{mg} / \mathrm{m}^{3} \mathrm{~d}$ (D, cooled): $4 \mathrm{M}$ 4F rats: $8 \times 6-\mathrm{hr}$ exposures: limp, eye and nose irritation, respiratory difficulty, weight loss: autopsy, organs normal

$100 \mathrm{mg} / \mathrm{m}^{3}$ d (D, cooled): $4 \mathrm{M} 4 \mathrm{~F}$ rats: $15 \times 6-\mathrm{hr}$ exposures: eye and nose irritation, respiratory difficulty, lethargy: autopsy, organs normal

$33 \mathrm{mg} / \mathrm{m}^{3} \mathrm{~d}$ (D, pet. ether, cooled): $15 \times 6$-hr expo- 
sures: $4 \mathrm{M}$ 4F rats: no toxic signs: autopsy, organs normal

Trichloromethylsulphenyl chloride liq. b.p. $148-9^{\circ} \mathrm{C}$

$100 \mathrm{ppm}$ (D): $4 \mathrm{M}$ rats: $1 \times 1-\mathrm{hr}$ exposure: severe respiratory difficulty, all died: autopsy (histol.) lung oedema

$10 \mathrm{ppm}(\mathrm{D}$, acetone): $4 \mathrm{M}$ rats: $1 \times 6-\mathrm{hr}$ exposure: lethargy, respiratory difficulty, 3 died later: autopsy (histol.) lung oedema

$2 \mathrm{ppm}$ (D, acetone): $4 \mathrm{M}$ rats: $20 \times 6-\mathrm{hr}$ exposures: initial respiratory difficulty: autopsy, lungs congested

$0.5 \mathrm{ppm}$ (D, acetone): $4 \mathrm{M} 4 \mathrm{~F}$ rats: $20 \times 6-\mathrm{hr}$ exposures: no toxic signs: autopsy, organs normal

Dimethyl disulphide

liq. b.p. $112^{\circ} \mathrm{C}$

$250 \mathrm{ppm}$ (D): $2 \mathrm{M} 2 \mathrm{~F}$ rats: $13 \times 6-\mathrm{hr}$ exposures: lethargy, respiratory difficulty, low weight gain: autopsy, organs congested

$100 \mathrm{ppm}(\mathrm{D}): 2 \mathrm{M} 2 \mathrm{~F}$ rats: $20 \times 6-\mathrm{hr}$ exposures: no toxic signs: autopsy, organs normal

Dixylyl disulphide (solution in mineral oil contained about $40 \%$ with other sulphides)

Saturated [3.5 2 /litre]: $4 \mathrm{~F}$ rats: $\left.15 \times \mathrm{Me}_{2} \mathrm{C}_{6} \mathrm{H}_{3} . \mathrm{S}\right]_{2}$ no toxic signs: autopsy, organs normal

Divinyl disulphide

liq. b.p. $86^{\circ} \mathrm{C}$

$480 \mathrm{ppm}$ (D): $2 \mathrm{M} 2 \mathrm{~F}$ rats: $1 \times 4 \cdot 5-\mathrm{hr}$ exposure: eye and nose irritation, respiratory difficulty, weight loss, 1 rat died later: autopsy (histol.) livers congested with necrosis and fibrosis

$130 \mathrm{ppm}$ (D, acetone): $2 \mathrm{M} 2 \mathrm{~F}$ rats: $4 \times 5$-hr exposures: intense lachrymation, nose irritation, lethargy, weight loss: autopsy, organs normal

$18 \mathrm{ppm}$ (D, acetone): $2 \mathrm{M} 2 \mathrm{~F}$ rats: $15 \times 6$-hr exposures: initial nose and eye irritation, lethargy, poor condition, retarded weight gain, blood and urine tests normal: autopsy, organs normal

$6 \mathrm{ppm}$ (D, acetone): $2 \mathrm{M} 2 \mathrm{~F}$ rats: $15 \times 6-\mathrm{hr}$ exposures: no toxic signs: autopsy, organs normal

Sulphur chloride pentafluoride

vapour b.p. $-20^{\circ} \mathrm{C}$

$100 \mathrm{ppm}(\mathrm{F}): 2 \mathrm{M}$ rats: $1 \times 1-\mathrm{hr}$ exposure: severe respiratory difficulty, both died: autopsy, lungs swollen and dark, (histol.) lungs-oedema and haemorrhage, liver and kidneys-congestion

$20 \mathrm{ppm}(\mathrm{F}): 2 \mathrm{M} 2 \mathrm{~F}$ rats: $1 \times 5-\mathrm{hr}$ exposure: respiratory difficulty: autopsy (histol.) lungs-oedema and congestion, liver and kidneys-congestion

$5 \mathrm{ppm}(\mathrm{F}): 4 \mathrm{M}$ 4F rats: $3 \times 5$-hr exposures: respiratory difficulty, weight loss: autopsy (histol.) lungscongestion and oedema

$1 \mathrm{ppm}(\mathrm{F}): 4 \mathrm{M}$ 4F rats: $20 \times 6-\mathrm{hr}$ exposures: no toxic signs: autopsy, organs normal

Bispentafluorosulphur oxide

liq. b.p. $29^{\circ} \mathrm{C}$

$\mathrm{S}_{2} \mathrm{~F}_{10} \mathrm{O}$
$1000 \mathrm{ppm}(\mathrm{D}$, cooled): $4 \mathrm{~F}$ rats: $1 \times 2-\mathrm{hr}$ exposure: respiratory difficulty, narcosis, cyanosis, froth at nose, all died: autopsy, severe lung oedema

$100 \mathrm{ppm}(\mathrm{D}$, cooled): $4 \mathrm{~F}$ rats: $1 \times 2 \cdot 5-\mathrm{hr}$ exposure: respiratory difficulty, narcosis, cyanosis, all died: autopsy, severe lung oedema

$20 \mathrm{ppm}$ (D, pet. ether): $4 \mathrm{~F}$ rats: $1 \times 6-\mathrm{hr}$ exposure: no toxic signs during exposure, 2 died later: autopsy (histol.) marked perivascular and peribronchiolar lung oedema, acute bronchitis

10 ppm (D, pet. ether): 4M 4F rats: $20 \times 6$-hr exposures: lethargy, respiratory difficulty, weight gain retarded: autopsy (histol.) lung oedema, liver, kidneys and spleen congested

$5 \mathrm{ppm}$ (D, pet. ether): $4 \mathrm{M} 4 \mathrm{~F}$ rats: $20 \times 6$-hr exposures: no toxic signs: autopsy, organs normal

Lauryl mercaptan

$\mathrm{C}_{12} \mathrm{H}_{25} \cdot \mathrm{SH}$

[dodecanethiol]

liq. b.p. $144-148^{\circ} \mathrm{C}(95 \mathrm{~mm})$

Saturated (A): $2 \mathrm{M}$ 2F rats: $20 \times 6-\mathrm{hr}$ exposures: no toxic signs: autopsy, organs normal

Vinylsulphur pentafluoride

liq. b.p. $41^{\circ} \mathrm{C}$

$800 \mathrm{ppm}(\mathrm{D}$, cooled): $2 \mathrm{M} 2 \mathrm{~F}$ rats: $1 \times 5$-hr exposure: respiratory difficulty, lethargy, incoordination, 1 died: autopsy (histol.) livers-inflammation and fatty infiltration, kidneys-tubules dilated with degeneration

200 ppm (D, cooled): $2 \mathrm{M} 2 \mathrm{~F}$ rats: $5 \times 6$-hr exposures: respiratory difficulty, lethargy weight loss, 1 died: autopsy (histol.) lungs-congestion and inflammation, livers-congestion and fatty infiltration, kidneys-tubules dilated with some degeneration

$50 \mathrm{ppm}(\mathrm{D}$, cooled): $4 \mathrm{M} 4 \mathrm{~F}$ rats: $19 \times 6$-hr exposures: no toxic signs: autopsy, organs normal

2-Chloroethylsulphur pentafluoride $\quad \mathrm{Cl} \cdot \mathrm{CH}_{2} \cdot \mathrm{CH}_{2} \cdot \mathrm{SF}_{5}$ liq. b.p. $92^{\circ} \mathrm{C}$

$200 \mathrm{ppm}(\mathrm{D}): 4 \mathrm{M}$ rats: $1 \times 2-\mathrm{hr}$ exposure: tremors and convulsions, 1 died: autopsy, organs normal

$50 \mathrm{ppm}$ (D): $4 \mathrm{M}$ 4F rats: $20 \times 6$-hr exposures: no toxic signs: autopsy, organs normal

2-Chlorotetrafluoroethylsulphur pentafluoride

liq. b.p. $46 \cdot 5^{\circ} \mathrm{C}$

$\mathrm{Cl} \cdot \mathrm{CF}_{2} \cdot \mathrm{CF}_{2} \cdot \mathrm{SF}_{8}$

$5000 \mathrm{ppm}$ (D, cooled): $2 \mathrm{M} 2 \mathrm{~F}$ rats: $1 \times 3-\mathrm{hl}$ exposure: respiratory difficulty leading to gasping and convulsions, all died during or soon after exposure: autopsy (histol.) lungs congested and oedematous, liver congested

$1000 \mathrm{ppm}(\mathrm{D}$, cooled): $2 \mathrm{M} 2 \mathrm{~F}$ rats: $1 \times 6-\mathrm{hr}$ exposure: respiratory difficulty, 3 died after exposure: autopsy (histol.) lungs oedematous

$200 \mathrm{ppm}$ (D, cooled): $2 \mathrm{M} 2 \mathrm{~F}$ rats: $15 \times 6-\mathrm{hr}$ exposures: no toxic signs: autopsy, organs noimal

4-Chloro-octafluorobutyl sulphur pentafluoride

liq. b.p. $99^{\circ} \mathrm{C}$

$\mathrm{Cl}\left(\mathrm{CF}_{2}\right)_{4} \mathrm{SF}_{\text {s }}$ 
$500 \mathrm{ppm}$ (D): $3 \mathrm{~F}$ rats: $15 \times 6$-hr exposures: no toxic signs: autopsy, organs normal (slight lung congestion?)

6-Chlorododecafluorohexylsulphur pentafluoride

liq. b.p. $143^{\circ} \mathrm{C}$

$\mathrm{Cl}\left(\mathrm{CF}_{2}\right)_{6} \cdot \mathrm{SF}_{0}$

$2500 \mathrm{ppm}$ (D): $4 \mathrm{~F}$ rats: $2 \times 5$-hi exposures: no weight gain: autopsy, organs normal

$500 \mathrm{ppm}(\mathrm{D}): 4 \mathrm{~F}$ rats: $13 \times 6-\mathrm{hr}$ exposures: no toxic signs: autopsy, organs normal

\section{Miscellaneous}

$N N$-Hexamethyleneadipamide solid, m.p. $250^{\circ} \mathrm{C}$

Fume $18 \mathrm{mg} /$ litre $(\mathrm{H}): 4 \mathrm{M}$ $4 \mathrm{~F}$ rats: $15 \times 6$-hr exposures: no toxic signs: blood and urine tests normal: autopsy (histol.) liver cell vacuolation and

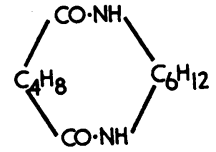
necrosis

Fume $15 \mathrm{mg} /$ litre $(\mathrm{H}): 4 \mathrm{M}$ 4F rats: $15 \times 6-\mathrm{hr}$ exposures: no toxic signs: autopsy, organs normal

Acrylyl chloride

[propenoyl chloride]

liq. b.p. $73^{\circ} \mathrm{C}$

$100 \mathrm{ppm}(\mathrm{D}): 4 \mathrm{M}$ rats: $1 \times 2-\mathrm{hr}$ exposure: lethargy, respiratory difficulty, autopsy, lung oedema

$25 \mathrm{ppm}(\mathrm{D}$, xylene): 4F rats: $1 \times 4$-hr exposure: eye irritation, respiratory difficulty, incoordination, 1 died: autopsy, lungs distended and oedematous, (histol.) lung emphysema and oedema

$5 \mathrm{ppm}(\mathrm{D}$, acetone): $4 \mathrm{~F}$ rats: $5 \times 5$-hr exposures: eye irritation, respiratory difficulty, lethargy, low rectal temperature, weight loss, 3 rats died on 3rd day: autopsy (histol.) pneumonia

$2.5 \mathrm{ppm}(\mathrm{D}$, acetone): $4 \mathrm{M}$ 4F rats: $3 \times 6-\mathrm{hr}$ exposures: weight loss, low rectal temperature, 1 died: autopsy, lungs distended, (histol.) lung oedema and inflammation

$1 \mathrm{ppm}(\mathrm{D}$, acetone): 4M 4F rats: $15 \times 6$-hr exposures: no toxic signs: autopsy, organs normal

2,2-Bis-p-hydroxyphenylpropane $\quad \mathrm{Me}_{2} \mathrm{C}\left(\mathrm{C}_{6} \mathrm{H}_{4} \cdot \mathrm{OH}-p\right)_{2}$ solid, m.p. $152-156^{\circ} \mathrm{C}$

Saturated (B): $4 M$ rats: $5 \times 6-\mathrm{hr}$ exposures: no toxic signs: autopsy, organs normal

Ethyl 3-chlorophenylformimidate $3-\mathrm{ClC}_{6} \mathrm{H}_{4} \cdot \mathrm{N}: \mathrm{CH} \cdot \mathrm{OEt}$ liq. b.p. $120^{\circ} \mathrm{C}(15 \mathrm{~mm})$

Saturated (A) $[0.26 \mathrm{mg} /$ litre, $33 \mathrm{ppm}]: 3 \mathrm{M}$ rats: $9 \times 6$-hr exposures: nose and eye irritation, lethargy, respiratory difficulty, weight loss: autopsy, lungs discoloured, (histol.) lungs - areas of consolidation and collapse with peribronchiolar lymphocytic reaction

$22 \mathrm{ppm}$ (D, ethanol): $6 \mathrm{M}$ rats: $11 \times 6$-hr exposures: slight lethargy and respiratory difficulty: autopsy, organs normal
Trimethoxyboroxine

liq. decomp.

Saturated (A) $[3 \mathrm{mg} / \mathrm{litre}$, $600 \mathrm{ppm}$ ]: $3 \mathrm{M} 4 \mathrm{~F}$ rats: $9 \times 6-\mathrm{hr}$ exposures:y slight lethargy: autops, organs normal

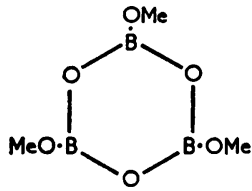

$N$-Propylethylideneamine

liq. b.p. $74^{\circ} \mathrm{C}$

$\mathrm{CH}_{3} \cdot \mathrm{CH}: \mathrm{N} \cdot \mathrm{C}_{3} \mathrm{H}_{2}$

$250 \mathrm{ppm}$ (D): $4 \mathrm{M}$ 4F rats: $1 \times 5-\mathrm{hr}$ exposure: eye and nose irritation, respiratory difficulty $(M$ more affected), poor condition: autopsy (histol.) increased macrophages in lungs

$100 \mathrm{ppm}(\mathrm{D}): 4 \mathrm{M}$ 4F rats: $6 \times 6$-hr exposures: nose irritation, respiratory difficulty, lethargy, weight loss, 1 died, blood and urine tests normal: autopsy (histol.) increased macrophages in lungs

$10 \mathrm{ppm}(\mathrm{D}$, pet. ether): $4 \mathrm{M}$ 4F rats: $15 \times 6-\mathrm{hr}$ exposures: no toxic signs apart from retarded weight gain, blood and urine tests normal: autopsy, organs normal

5 ppm (D, pet. ether): $4 \mathrm{M}$ 4F rats: $15 \times 6-\mathrm{hr}$ exposures: no toxic signs: autopsy, organs normal

Iron pentacarbonyl

$\mathrm{Fe}(\mathrm{CO})_{5}$

liq. b.p. $103^{\circ} \mathrm{C}$

$33 \mathrm{ppm}$ (D, pet. ether): $4 \mathrm{M}$ 4F rats: $1 \times 5 \cdot 5-\mathrm{hr}$ exposure: lethargy, respiratory difficulty, $4 \%$ carboxyhaemoglobin, 3 dead next day: autopsy (histol.) lung oedema and congestion

$15 \mathrm{ppm}$ (D, pet. ether): 4M 4F rats: $2 \times 5 \cdot 5-\mathrm{hr}$ exposures: lethargy, respiratory difficulty, $0 \cdot 2-0 \cdot 4 \%$ carboxyhaemoglobin, 4 dead 3-4 days later:autopsy (histol.) lung oedema and congestion

$7 \mathrm{ppm}$ (D, pet. ether): 4M 4F rats: $18 \times 5 \cdot 5-\mathrm{hr}$ exposures: no toxic signs: autopsy, organs normal

\section{Discussion}

\section{Review of toxicological properties}

Hydrocarbons None of the compounds examined showed any effects on the blood cells; the results with trimethylbenzene did not confirm the rather doubtful evidence that this compound has such an effect in man (Bättig, Grandjean, Rossi, and Rickenbacher, 1958). Soviet work (Korbakova, 1964) claims that low concentrations of cyclopentadiene and dicyclopentadiene can affect the blood as well as the lung and the functioning of the nervous system. Other Soviet work casts doubt on the low toxicity of isoprene, indicating marginal organ damage after prolonged exposure to $67-200 \mathrm{ppm}$ (Korbakova and Fedorova, 1964).

Alcohols The alcohols examined show interesting features. t-Butoxyethanol had the characteristic haemolytic action of $n$-butoxyethanol described by Werner, Nawrocki, Mitchell, Miller, and von Oettingen (1943), and Carpenter, Smyth, and Pozzani (1949). The results strongly suggest that the alcohol 
or its metabolite (Carpenter, Pozzani, Weil, Nair, Keck, and Smyth, 1956) increases the fragility of aged red cells, and that the effect disappears when the average age is reduced by haemopoiesis, returning only when the normal age distribution is restored. Isopropoxyethanol shows the same effect but to a lesser extent. Perfluoro-triethylcarbinol is a powerful uncoupler of oxidative phosphorylation, an effect which has been confirmed by in vitro studies on mitochondria in which a dissociation between oxygen uptake and the phosphorylation of ADP was observed which was quantitatively and qualitatively similar to that from dinitrophenol (Gage, unpublished work). This compound must be one of the simplest to show this effect; it conforms to the requirements of an uncoupler (Hemker, 1962) in that it is acidic due to the influence of the fluorine atoms on the hydroxyl group, and it is lipidsoluble in its non-ionized form. Chloropropanol is irritant but it does not show the central effects of chloroethanol (Goldblatt and Chiesman, 1944).

Ethers All the ethers had the central depressant action of diethyl ether, with the exception of dichlorodiisopropyl ether, which showed irritant properties, like its ethyl homologue (American Petroleum Institute, 1948).

Aldehydes and ketones The aldehydes have anaesthetic properties, with no marked irritant action. According to Skog (1950), the $\mathrm{LC}_{50}$ (30 minutes) of butyraldehyde to rats is $6 \% \mathrm{v} / \mathrm{v}$. Sim and Pattle (1957) state that groups of men exposed to concentrations of butyraldehyde and isobutyraldehyde greater than $200 \mathrm{ppm}$ for $\mathbf{3 0}$ minutes experienced no irritation, but some nausea with isobutyraldehyde. Soviet investigations on man (Uloyan, 1965) claim that a variety of effects are produced at $3 \mathrm{ppm}$.

Acids A comparison of the results with acrylic and methacrylic acids demonstrates the reduction in irritant action by the introduction of a methyl group, an effect observed with the methyl esters which show a 10-fold difference in American Conference of Governmental Industrial Hygienists (ACGIH, 1968) threshold limit values. The low toxicity of methacrylic acid in animals is in conflict with Soviet claims (Stulova, Rumyantseva, and Ivanova, 1962) that concentrations down to $6 \mathrm{ppm}$ produce marginal changes in the function of the nervous system in man.

Chlorinated hydrocarbons The aliphatic chlorinated hydrocarbons demonstrated the liver and kidney damage characteristic of some members of this series. The two unsaturated 4-carbon compounds, dichlorobutene and hexachlorobutadiene, are highly toxic; both are capable of producing severe kidney damage, but with dichlorobutene lung irritation predominates and the renal effect has been observed only after percutaneous absorption (Ferguson, unpublished work). Smyth, Carpenter, and Weil (1951) found that exposure of rats for 4 hours to $62 \mathrm{ppm}$ dichlorobutene (isomer unspecified) killed $2 / 6$. The chlorinated ethylenes, on the other hand, are of relatively low toxicity. The results with 1,1dichloroethene are extended by the observations of Prendergast, Jones, Jenkins, and Siegel (1967), who found no clear toxic manifestations apart from a retarded weight increase in a variety of species exposed to $100 \mathrm{ppm} 5$ hours/day for six weeks, while some liver damage was found after continuous exposures 24 hours/day to $47 \mathrm{ppm}$ for 90 days. Rylova (1953) states that $25 \mathrm{ppm}$ is irritant to man.

Pentafluorobenzene is a narcotic; Garmer and Leigh (1967) have shown that the anaesthetic concentration for cats is $1.5-2.5 \% \mathrm{v} / \mathrm{v}$. As the fluorine atoms are replaced by chlorine, the anaesthetic action remains but a cytotoxic action appears, together with an effect on porphyrin metabolism.

The results with chloronaphthalene are in contradiction to Soviet claims (Kapkaev, 1957) that concentrations in the region of $1 \mathrm{ppm}$ cause liver damage with a variety of blood changes and a hyperacidic gastritis.

Esters The unsaturated esters of saturated carboxylic acids are typically of low toxicity, high concentrations producing irritation and narcosis. With the exception of vinyl acetate, none of the esters of this type which have been examined was sufficiently volatile to show these effects. Dimethyl carbonate rapidly hydrolyses so its toxicity may be taken to be that of methanol. The high toxicity of the chloroformates is due to their great chemical reactivity; presumably like phosgene they modify cell membranes to produce permeability changes. Methyl nitrite produced methaemoglobinaemia in vivo at a rate similar to that of sodium nitrite; if its action is due to inorganic nitrite then its hydrolysis in vivo must be very rapid.

Amines The amines examined showed irritant and central stimulant effects; these increased with the degree of substitution, but the higher members have too low a volatility to present a significant vapour hazard. The results with diaminohexane are in contradiction to Soviet claims (Kulakov, 1967) that changes in the blood cells and in motor responses are seen in rats at $0.04 \mathrm{mg} / \mathrm{m}^{3}$.

Nitriles The compounds tested were primarily irritant, and there is no clear indication that any of the effects observed were due to liberated cyanide.

Heterocyclics There are no notable common features in this group. 
Organic peroxides These compounds were irritant to the eyes and respiratory tract, presumably because of their high reactivity, but it is not possible to relate their toxicity to their reactivity with iodide solution. The results obtained with cumene hydroperoxide may be compared with those of Floyd and Stokinger (1958), who found the $\mathrm{LC}_{50}$ (4 hours) to be $220 \mathrm{ppm}$ in rats. Soviet claims (Solomin, 1964, 1966) suggest that the concentration of this compound must be reduced to $0.001 \mathrm{ppm}$ to avoid effects on animals and on man.

Organic phosphorus compounds Trishydroxymethylpropane phosphite has shown an unexpectedly high toxicity: it is one of the most toxic compounds handled in this laboratory. It is fairly readily hydrolysed to yield dihydroxybutylphosphonic acid, which is of low toxicity by oral or parenteral administration. Its marked action on the central nervous system is probably due to its having sufficient stability to penetrate cell membranes as a non-ionized molecule, and its ultimate action may be due to its hydrolysis product or a reaction in situ.

Diethyl phosphorchloridothionate is primarily an irritant, presumably due to the reactive chlorine atom, but it is also a weak in vivo inhibitor of cholinesterase.

Silicon compounds The toxicity of silicon tetrafluoride is probably due to hydrogen fluoride released by hydrolysis. Similarly, the irritant action of silicon tetraisocyanate may be due to isocyanic acid. The stable silane derivatives are of low toxicity.

Sulphur compounds This group contains members which, presumably because of their high reactivity, are powerful lung irritants. Sulphur chloride pentafluoride, bispentafluorosulphur monoxide, and trichloro-methylsulphenyl chloride are at least as toxic as phosgene. The toxicity of sulphur dichloride is probably due to hydrolysis to hydrogen chloride. Vinylsulphur pentafluoride and divinyl disulphide have, in addition to their irritant action, a toxic effect on the liver or kidneys.

Miscellaneous Iron pentacarbonyl is a lung irritant, it affects the central nervous system and causes liver and kidney damage. A measurement of blood carboxyhaemoglobin would provide no guide to intoxication.

\section{The value of inhalation experiments}

Most of the substances which have been tested in these inhalation experiments have also been studied in these laboratories for oral and parenteral toxicity and for their effects on the skin and eyes. The systemic effects elicited after oral or intraperitoneal administration, or by percutaneous absorption, give, in general, a qualitative indication of the systemic effects obtained by inhalation studies, but, because of the differences in rates of absorption and metabolic transformations, there is little useful quantitative information.

Inhalation experiments are of special value for the study of those compounds which have an immediate or delayed irritant action on the lungs, for the intensity of these effects cannot be predicted with any certainty by other routes of administration. A survey of the results gives a strong indication that these effects on the lung are associated with the chemical reactivity of the molecule, particularly if the onset of severe symptoms is delayed. It seems probable that there is an initial modification of cell membranes, as postulated for the action of phosgene and ketene, followed by permeability changes leading to oedema and haemorrhage.

After short exposures to high concentrations of lung iritants, the effects seen at histological examination of lung tissue indicate that death can be attributed to an interference with gas exchange. After more prolonged exposure to lower concentrations, the cause of death is less certain, for although lung changes may be apparent, they are sometimes insufficient to account for the lethal action. Moreover, at still lower concentrations the animals may be in poor condition with a diminished weight increase, without any trace of damage being detectable in the lungs. It seems likely that exposure to irritant gases and vapours gives rise to stress which is responsible for the marginal toxic effects, and it is possible that the occasional observation of a diminution in the size of the thymus may be in some way connected with such stress effects.

\section{Provisional operational limits}

Subacute inhalation experiments lasting approximately three weeks cannot be regarded as an adequate basis for the establishment of threshold limit values which will define safe working concentrations under all conditions, although a study of the origins of the list published by the ACGIH (1966) shows that some of their values have been derived from more tenuous evidence. Nevertheless, the results obtained in these investigations permit an assessment of the toxic hazard which is of value to the chemical engineer in the design of plant, or which can form the basis of a code of safety precautions, provided that those exposed are under adequate medical supervision. The limiting concentrations derived from these experiments may be termed provisional operational limits to distinguish them from threshold limit values, which should preferably be based on more extended experiments on a variety of species, supported by evidence from human exposure.

The limits in Table 1 have been derived from the 
highest concentration producing no toxic effects in animals by the application of a 'safety factor', which has varied according to the effects seen at the next highest concentration. In some cases the limit has been influenced by the established threshold limit values of analogous substances, and any information on human exposure has been taken into consideration. With materials with a strong odour, the limit has been set at the expected tolerable level.

Not all of the substances studied have proved to be of commercial interest. Those which have been introduced into manufacturing processes have been handled under proper supervision and there is no indication that the provisional operational limit has been set too high.

\section{Comparison with Soviet limits}

None of the substances studied has yet been considered by the ACGIH Committee on Threshold Limit Values (1968), but several have been the subject of investigations on animals and on man in the Soviet Union, which have led to the recommended maximal allowable concentrations in Table 2. The differences between the limits in Tables 1 and 2 are too large to be ignored. The Soviet animal experiments are usually of several months' duration, but it is unlikely that this plays an important part as there is often a wide divergence between the ACGIH threshold limit values and Soviet maximal allowable concentrations, even when the exposure periods are similar. The Soviet results cannot be passed over by doubting the toxicological significance of studies on nervous system function by techniques such as the conditioned reflex, negative induction or electroencephalography. It is true that these methods are extensively used and that there is little information in the English language dealing with the experimental details and the interpretation of the results; the review by Medved and Kagan (1966) indicates that many of the original publications are in journals inaccessible outside the Soviet Union. It is more disturbing that the Soviet investigators appear to have far more sensitive indices of early haematological changes and of organ damage. In the few Russian papers available to the author in translation, there is little indication how the statistical significance of differences between the test animals and a control group under identical conditions has been established. There is no doubt of the need for closer collaboration between toxicologists inside and outside the Soviet Union.

I wish to acknowledge the skilled assistance which I have received over the years, particularly from $\mathrm{Mr}$. Z. S. Berczy, Mr. C. A. Manley, and Mr. R. A. Riley. Pathological reports have been prepared by Dr. J. G. S. Crabbe, Dr. E. Weston Hurst, Dr. T. F. McElligott, and Dr. D. M. Conning.
TABLE 1

\section{Provisional Operational Limits}

(Figures are ppm unless otherwise stated)

\begin{tabular}{|c|c|c|c|c|}
\hline Isoprene & $\cdots$ & .. & $\cdots$ & \\
\hline Dicyclopentadiene & . & . & . & 20 \\
\hline Decalin $\quad \ldots$ & . & . & . & 25 \\
\hline 1,2,4-Trimethylbenzene & & .. & . & 100 \\
\hline 2-Isopropoxyethanol & . & . & . & 50 \\
\hline 2-t-Butoxyethanol & . & . & . & 10 \\
\hline Chloropropanol & & & .. & 10 \\
\hline Tris(pentafluoroethyl)m & nethanc & & . & $0 \cdot 2$ \\
\hline Dichlorodi-isopropyl et & ther & .. & . & 15 \\
\hline Diethyleneglycol dimet & thyl eth & & . & 100 \\
\hline Methyl vinyl ether & $\cdots$ & . & .. & 400 \\
\hline Isobutyl vinyl ether & . & .. & . & 100 \\
\hline Dimethoxymethane & .. & . & . & 1000 \\
\hline Propionaldehyde & . & . & . & 200 \\
\hline n-Butyraldehyde & .. & .. & . & 200 \\
\hline Isobutyraldehyde & . & .. & .. & 100 \\
\hline 5-Bromopentan-2-one & . & . & . & 10 \\
\hline 2-Chloropropane & $\cdots$ & . & . & 50 \\
\hline 1,1-Dichloroethene & . & . & . & 25 \\
\hline Dichlorobutenes & . & .. & .. & $0 \cdot 1$ \\
\hline Hexachlorobutadiene & . & . & . & 1 \\
\hline 1,2,4-Trichlorobenzene & & .. & . & 25 \\
\hline Hexafluorobenzene & . & . & . & 100 \\
\hline Chloropentafluorobenz & rene & .. & .. & 100 \\
\hline 1,3,5-Trichlorotrifluoro & benzen & & . & 25 \\
\hline Acrylic acid $\quad \ldots$ & . & .. & .. & 20 \\
\hline Methacrylic acid & . & .. & .. & 20 \\
\hline Methyl nitrite & . & . & . & 10 \\
\hline Dimethyl carbonate & .. & .. & .. & 100 \\
\hline Vinyl acetate $\quad .$. & . & . & .. & 50 \\
\hline Methyl isothiocyanate & & . & .. & 1 \\
\hline 2-Ethylhexyl acrylate & & .. & .. & 50 \\
\hline 2-Ethylhexyl methacryl & & .. & .. & 25 \\
\hline Methyl chloroformate & & $\ldots$ & .. & 1 \\
\hline Ethyl chloroformate & . & . & .. & 1 \\
\hline Isopropyl chloroformat & & .. & .. & 2 \\
\hline s-Butylamine ... & . & .. & .. & 75 \\
\hline Di-s-butylamine & . & .. & .. & 25 \\
\hline Tributylamine $\ldots$ & . & .. & . & 10 \\
\hline Nonylamine $\quad \ldots$ & . & .. & .. & 10 \\
\hline Aminobutanol & .. & .. & .. & 25 \\
\hline 1,6-Diaminohexane & . & . & .. & 25 \\
\hline Diethylaminopentan-2- & one & .. & .. & 25 \\
\hline Propyl cyanide ... & .. & . & .. & 50 \\
\hline Chloroacetonitrile & . & . & .. & 5 \\
\hline 2-Methoxy-2,3-dihydro & pyran & & .. & 100 \\
\hline 2-Aminomethyl-3,4-dih & ydropy & yran & . & 5 \\
\hline 2-Methylbenzoxazole & . & . & . & 50 \\
\hline$N$-Formylpiperidine & . & .. & .. & 50 \\
\hline 2-Methylthiazole & . & $\ldots$ & .. & 10 \\
\hline 3,5-Dimethylmorpholin & & .. & .. & 25 \\
\hline 2-Methyl-1,3-dioxolane & & .. & .. & 50 \\
\hline t-Butyl peroxypivalate & & . & .. & 20 \\
\hline Ethyl t-butyl peroxyox & alate & & .. & 25 \\
\hline Dipropionyl peroxide & & . & .. & 2 \\
\hline Diiso-amyl peroxydicar & rbonate & & .. & $10 \mathrm{mg} / \mathrm{m}^{3}$ \\
\hline Cumene hydroperoxide & & . & .. & 10 \\
\hline t-Butyl peracetate & & . & . & 0.05 \\
\hline Trimethylolpropane ph & osphite & & .. & $0 \cdot 1$ \\
\hline Phosphorus triisocyana & ite & .. & . & 2 \\
\hline Silicon tetrafluoride & . & .. & .. & 3 \\
\hline Silicon tetraisocyanate & .. & $\ldots$ & .. & 20 \\
\hline Tetramethylsilane & . & $\cdots$ & .. & 250 \\
\hline Sulphur dichloride & . & .. & .. & $5($ as $\mathrm{HCl})$ \\
\hline
\end{tabular}


TABLE 1 (continued)

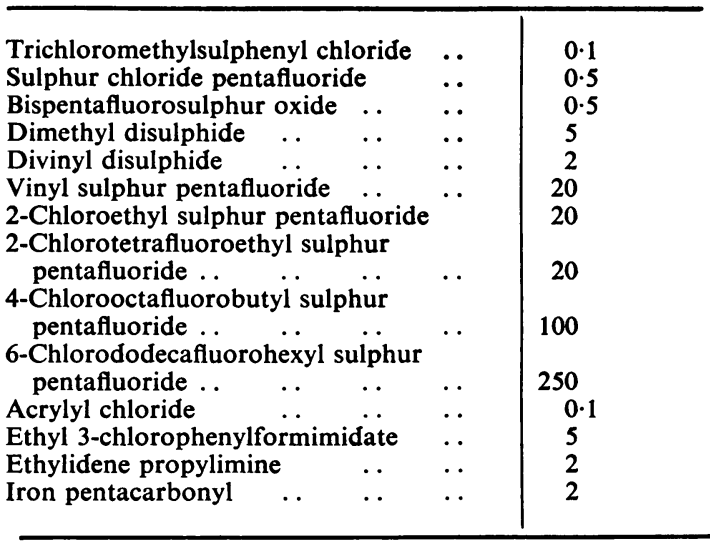

TABLE 2

\section{Soviet Recommended Maximal Allowable CONCENTRATIONS}

\begin{tabular}{|c|c|c|}
\hline & ppm & \\
\hline Isoprene & 17 & $\begin{array}{l}\text { Korbakova and Fedorova } \\
\text { (1964) }\end{array}$ \\
\hline $\begin{array}{l}\text { Cyclopentadiene } \\
\text { Methacrylic acid }\end{array}$ & $\begin{array}{l}2 \\
0.003\end{array}$ & $\begin{array}{l}\text { Uloyan (1965) } \\
\text { Stulova, Rumyantseva, and } \\
\text { Ivanova (1962) }\end{array}$ \\
\hline $\begin{array}{l}\text { Butyraldehyde } \\
\text { Diaminohexane } \\
\text { Cumene } \\
\text { hydroperoxide }\end{array}$ & $\begin{array}{l}0.3 \\
0.0002 \\
0.001\end{array}$ & $\begin{array}{l}\text { Korbakova (1964) } \\
\text { Kulakov (1967) } \\
\text { Solomin (1966) }\end{array}$ \\
\hline
\end{tabular}

\section{References}

American Conference of Governmental Industrial Hygienists (1966). Documentation of threshold limit values.

- (1968). Threshold limit values for air-borne contaminants.

American Petroleum Institute (1948). API Toxicological Reviews, $\beta, \beta^{\prime}$-Dichloroethyl ether.

Bättig, K., Grandjean, E., Rossi, L., and Rickenbacher, J. (1958). Toxikologische Untersuchungen über Trimethylbenzol. Arch. Gewerbepath. Gewerbehyg., 16, 555-566.

Byers, P. D., and Gage, J. C. (1961). The toxicity of precipitated silica. Brit. J. industr. Med., 18, 295-302.

Carpenter, C. P., Pozzani, U. C., Weil, C. S., Nair, J. H., Keck, G. A., and Smyth, H. F. Jr. (1956). The toxicity of butyl cellosolve solvent. Arch. industr. Hlth, 14, 114-131.
Carpenter, C. P., Smyth, H. F. Jr., and Pozzani, U. C. (1949). The assay of acute vapor toxicity, and the grading and interpretation of results on 96 chemical compounds. J. industr. Hyg., 31, 343-346.

Floyd, E. P., and Stokinger, H. E. (1958). Toxicity studies of certain organic peroxides and hydroperoxides. Amer. industr. Hyg. Ass. J., 19, 205-212.

Gage, J. C. (1953). A controlled fluid-feed atomizer. J. sci. Instrum., $30,25$.

(1959). The toxicity of epichlorohydrin vapour. Brit. J. industr. Med., 16, 11-14.

(1968). Toxicity of paraquat and diquat aerosols generated by a size-selective cyclone: effect of particle size distribution. Ibid., 25, 304-314.

Garmer, N. L., and Leigh, J. M. (1967). Some effects of hexafluorobenzene in cats. Brit. J. Pharmacol., 31, 345-350.

Goldblatt, M. W., and Chiesman, W. E. (1944). Toxic effects of ethylene chlorohydrin. Brit. J. industr. Med., 1, 207-223.

Hemker, H. C. (1962). Lipid solubility as a factor influencing the activity of uncoupling phenols. Biochim. biophys. Acta, 63, 46.

Kapkaev, A. (1957). Characteristics of Halowax as an industria poison. Quoted from Chem. Abstr., 1958, 52, 14001a.

Korbakova, A. I. (1964). Standard levels of new industrial chemicals in the air of work premises. Quoted from Chem. Abstr., 1964, 61, $16694 \mathrm{~b}$.

, and Fedorova, V. I. (1964). Toxicology of isoprene. Quoted from Chem. Abstr., 1965, 63, 17017a.

Kulakov, A. E. (1967). Permissible concentration of hexamethylenediamine in the air in populated areas. Quoted from Chem. Abstr., 1968, 69, p.1221, abstr. no. 12744h.

Medved, L. I., and Kagan, J. S. (1966). Toxicology Ann. Rev. Pharmacol., 6, 293-308.

Prendergast, J. A., Jones, R. A., Jenkins, L. J., and Siegel, J. (1967). Effects on experimental animals of long-term inhalation of trichloroethylene, carbon tetrachloride, 1,1,1-trichloroethane, dichlorodifluoro-methane and 1,1-dichloroethylene. Toxicol. appl. Pharmacol., 10, 270-289.

Rylova, M. L. (1953). Toxicity of 1,1-ethene dichloride. Quoted from Chem. Abstr., 1953, 47, 11559i.

Sim, V. M., and Pattle, R. E. (1957). Effect of possible smog irritants on human subjects. J. Amer. med. Ass., 165, 1908-1913.

Skog, E. (1950). A toxicological investigation of lower aliphatic aldehydes. I. Toxicity of formaldehyde, acetaldehyde, propionaldehyde and butyraldehyde; as well as of acrolein and crotonaldehyde. Acta pharmacol. (Kbh), 6, 299-318.

Smyth, H. F., Carpenter, C. P. and Weil, C. S. (1951). Range-finding toxicity data: list IV. Arch. industr. Hyg., 4, 119-122.

Solomin, G. I. (1964). Maximum permissible concentration of isopropylbenzene and its hidroperoxide in the atmosphere. Quoted from Chem. Abstr., 1964, 60, 13782 f.

- (1966). Hygienic substantiation of the daily average maximum permissible concentration of isopropylbenzene and its hydroperoxide in the atmosphere. Quoted from Chem. Abstr. $1966,65,4523 d$.

Stulova, E. A., Rumyantseva, E. P., and Ivanova, A. G. (1962). Influence of methacrylic acid on the health of workers. Quoted from Chem. Abstr., 1964, 61, Column 8808b.

Uloyan, S. M. (1965). Permissible concentration of butyraldehyde in the air of industrial areas. Quoted from Chem. Abstr., 1966, $65,4524 h$.

Werner, H. W., Nawrocki, C. Z., Mitchell, J. L., Miller, J. W., and von Oettingen, W. F. (1943). Effects of repeated exposures of rats to vapors of monoalkyl ethylene glycol ethers. $J$. industr. Hyg., 25, 374-379.

Received for publication July 29, 1969. 